\title{
Hyphal compartmentalization and sporulation in Streptomyces require the conserved cell division protein SepX
}

\author{
Matthew J. Bush ${ }^{1}$, Kelley A. Gallagher ${ }^{1,3}$, Govind Chandra ${ }^{1}$, Kim C. Findlay ${ }^{2}$ and \\ Susan Schlimpert ${ }^{1 *}$
}

\section{Affiliations}

${ }^{1}$ Department of Molecular Microbiology, John Innes Centre, Norwich Research Park, Norwich NR47HU UK

${ }^{2}$ Department of Cell and Developmental Biology, John Innes Centre, Norwich Research Park, Norwich, NR4-7HU, UK

${ }^{3}$ Département de Microbiologie, Infectiologie et Immunologie, Université de Montréal, Pavillon Roger-Gaudry, 2900, boulevard Édouard-Montpetit, C.P. 6128, Succursale Centre-ville, Montréal, QC H3C 3J7, Canada

* Correspondence: susan.schlimpert@jic.ac.uk 


\begin{abstract}
Filamentous actinobacteria such as Streptomyces undergo two distinct modes of cell division, leading to partitioning of growing hyphae into multicellular compartments via cross-walls, and to septation and release of unicellular spores. Specific determinants for cross-wall formation and the importance of hyphal compartmentalization for Streptomyces development are largely unknown. Here we show that SepX, an actinobacterial-specific protein, is crucial for both cell division modes in Streptomyces venezuelae. Importantly, we find that sepX-deficient mutants grow without cross-walls and that this substantially impairs the fitness of colonies and the coordinated progression through the developmental life cycle. Protein interaction studies and live-cell imaging suggest that SepX contributes to the stabilization of the divisome, a mechanism that also requires the dynamin-like protein DynB. Thus, our work identifies an important determinant for cell division in Streptomyces that is required for cellular development and sporulation.
\end{abstract}




\section{Introduction}

Most bacteria divide by binary fission which results in the generation of two virtually identical daughter cells that physically separate to colonize new environmental niches. However, in filamentous growing organisms, such as the abundant soil bacteria of the genus Streptomyces, two functionally distinct modes of cell division exist: cross-wall formation in vegetative hyphae and sporulation in aerial hyphae (Figure 1a). Cross-walls are division septa that partition growing hyphae at irregular intervals into multinucleoid compartments, reminiscent of fungal hyphae. In contrast, sporulation-specific cell division results in the formation of ladder-like arrays of division septa that are structurally distinct from cross-walls, and which lead to cell fission and the release of unigenomic spores ${ }^{1}$. While sporulation is an important strategy for survival and propagation ${ }^{2,3}$, the physiological significance of cross-wall formation during vegetative growth is much less understood.

At the heart of both division processes is the almost universally conserved cell division protein FtsZ that assembles into a contractile ring-like structure called the Z-ring. The Z-ring acts as a scaffold for the assembly of the cell division machinery (the divisome) and guides the synthesis of septal peptidoglycan ${ }^{4,5}$. Streptomyces encode several orthologues of the core divisome components from E. coli or B. subtilis, such as FtsK, FtsQ, FtsL, DivIC and the cell wall synthetic enzymes Ftsl and FtsW ${ }^{6,7}$. Moreover, additional factors have been identified that associate with the divisome and/or contribute to the efficient assembly and stability of Zrings. These proteins include the actinobacterial-specific protein SsgB, which has been reported to mark future sporulation-septation sites ${ }^{8}, \mathrm{SepF}$, which is likely to function as a membrane anchor for $\mathrm{FtsZ}^{9-11}$, SepH, which stimulates FtsZ filament assembly ${ }^{12}$, the two dynamin-like proteins DynA and DynB, which stabilize FtsZ-rings during sporulation, and two SepF-like proteins of unknown function ${ }^{11}$.

Previous genetic studies have shown that none of the conserved and species-specific cell division proteins are essential for growth and viability in Streptomyces ${ }^{6,7}$. While the deletion of core cell division genes, including $f t s Q, f t s L$, divIC, ftsW or ftsl, appears to largely affect sporulation septation ${ }^{6,8,11-13}$, a Streptomyces $\Delta f t s Z$ mutant is both unable to sporulate and to compartmentalize the vegetative mycelium, resulting in a single-celled, branched mycelial network. Hence, to-date fts $Z$ is the only known determinant of cross-wall formation.

Here, we report the identification of SepX, a Streptomyces divisome component that is required for compartmentalization of vegetative hyphae and for normal sporulation in aerial hyphae. Besides $f t s Z$, sepX is the only other determinant of vegetative septation identified so far and we show that cross-wall formation is crucial for fitness and cellular development in Streptomyces. We further demonstrate that SepX also plays an important role in sporulation-specific cell division via a direct interaction with the bacterial dynamin-like protein DynB, which was previously shown to contribute to Z-ring stability. We find that while either of the proteins is dispensable for sporulation, deletion of both $\operatorname{sep} X$ and $d y n B$ impairs 
stable Z-ring formation and function, and consequently abolishes sporulation-specific cell division.

\section{RESULTS}

\section{SepX co-localizes with FtsZ at division septa}

In S. venezuelae, the initiation of sporulation-specific cell division is co-controlled by the WhiA and WhiB transcription factors ${ }^{14,15}$. Using chromatin-immunoprecipitation (ChIP)-seq in concert with microarray transcriptional profiling, we previously revealed the WhiABmediated activation of several genes encoding core members of the bacterial division machinery, including $f t s W, f t s K$ and $f t s Z$. WhiA and WhiB together control the expression of $\sim 240$ transcriptional units and we reasoned that other genes encoding key components of the Streptomyces division machinery might also be under WhiAB control. Therefore, we constructed various strains expressing fluorescently tagged WhiAB-target proteins, prioritizing those encoded by conserved and uncharacterized genes with a clear dependence upon WhiA and WhiB for their expression.

As part of this study, we examined the localization of a protein encoded by $v n z$ _ 14865 (now named SepX for "septal protein X"), a target of WhiAB-mediated activation (Supplementary Figure 1a). SepX is a putative 344-amino acid membrane protein that contains two largely unstructured regions predicted to reside in the cytoplasm (Figure 1B). Further bioinformatic analysis indicated that SepX does not share any homology with proteins of known function. To visualize its subcellular localization, we constructed a merodiploid strain in which sepX was fused to mcherry and expressed in trans from its native promoter from the ФBT1 integration site. This strain additionally carried a $P_{f t s z}-f t s Z$-ypet gene fusion, thereby allowing us to fluorescently label both vegetative and sporulation septa. Microscopic analysis of this dual-labelled strain by time-lapse microscopy revealed that SepX-mCherry frequently co-localizes with FtsZ at both vegetative cross-walls and sporulation septa (Figure 1c, d, Supplementary Figure $1 \mathrm{~b}$ and Supplementary Movie 1). Control experiments using single-labelled strains confirmed that the detected fluorescent signals for FtsZ-YPet and SepXmCherry were specific (Supplementary Figure 1c). We were unable to resolve whether SepX localization precedes the arrival of FtsZ, due to the weak fluorescent signal of the SepXmCherry fusion when produced from its native promoter. The presence of SepX at both cell division sites is in line with transcriptomics data that showed expression of the sepX gene both during vegetative and reproductive growth. Furthermore, albeit at reduced levels, sepX transcripts were also detected in the absence of WhiA or WhiB (Supplementary Figure 1d), indicating that expression of $\operatorname{sep} X$ might be controlled by an additional factor.

To determine if SepX accumulation at nascent division sites was dependent on FtsZ, we inserted the sepX-mcherry fusion into the $\Delta$ ftsZ null mutant in trans. SepX-mCherry was produced from the constitutive ermE* promoter to ensure expression in the mutant 
background. Subsequently, we inspected hyphae of the resulting strain by fluorescence microscopy. In addition, we used the fluorescent D-amino acid analogue HADA to label cell wall peptidoglycan, including cross-walls and sporulation septa ${ }^{16}$. As expected, ftsZ-deficient hyphae were devoid of division septa. In these continuous hyphae, SepX-mCherry was stably produced and appeared to be distributed evenly within the hyphae, indicating that the distinct localization pattern of SepX-mCherry observed in the wildtype requires FtsZ (Supplementary Figure $1 \mathrm{e}$ and f).

\section{SepX is required for compartmentalization and sporulation}

Intrigued by the accumulation of SepX at both types of division septa, we engineered an inframe gene deletion and examined the importance of SepX for cell division during vegetative growth and sporulation. Using the fluorescent membrane dye FM4-64, we first visualized vegetative septa as a proxy for cross-walls in S. venezuelae, comparing the wildtype to the $\Delta$ sepX mutant. Strikingly, deletion of $\operatorname{sepX}$ led to a vegetative mycelium almost completely devoid of cross-walls (Figure $2 \mathrm{a}$ and $\mathrm{b}$ ). To better understand the effect of a sepX deletion on the overall frequency of cross-wall formation, we quantified the number of cross-walls produced in hyphae during vegetative growth. To achieve this, we allowed spores of the wildtype, the $\Delta \operatorname{sep} X$ mutant and the complemented mutant $\left(\Delta \operatorname{sep} X / \operatorname{sep} X^{+}\right)$to germinate and grow in the presence of HADA in a microfluidic device for five to six hours. We then imaged emerging hyphae by fluorescence microscopy and quantified the number of cross-walls per hypha. We found that in wild-type $S$. venezuelae cross-walls are synthesized on average every 20 to $30 \mu \mathrm{m}$ (Figure 2c). In agreement with the FM4-64 membrane staining (Figure 2b), deletion of sepX led to an almost total lack of cross-walls within the vegetative mycelium. Very occasionally, cross-wall formation occurred close to the "mother" spore, but even where such cross-walls were observed, the resulting vegetative mycelium was devoid of septa (Supplementary Figure 2a). The frequency of cross-wall formation was restored when $\Delta s e p X$ was complemented in trans.

Since a $\Delta$ sepX mutant lacks cross-walls, we wondered whether constitutive expression of sepX would lead to a converse increase in cross-wall frequency during vegetative growth. Therefore, we repeated our HADA labelling experiments with a strain that expressed sepX in trans from the constitutive ermE* promoter $^{17}$ (Supplementary Figure 2e). While we did not observe any visible effects on growth and sporulation of $S$. venezuelae (Supplementary Figure $2 \mathrm{~b})$, we found that approximately double the number of cross-walls could be observed in the $\mathrm{SepX}^{++}$strain compared to the wildtype, with a cross-wall forming every 10-15 $\mu \mathrm{m}$ (Supplementary Figure $2 \mathrm{c}$ and $\mathrm{d}$ ). Constitutive expression of other divisome components that are also part of the division machinery involved in vegetative cell division and cross-wall formation, including sepF and ftsZ itself, did not lead to an increase in cross-wall frequency (Supplementary Figure $2 c$ and $d$ ). Thus, these results indicate that SepX abundance is a 
determinant for FtsZ-dependent vegetative cell division leading to hyphal compartmentalization.

Notably, our membrane staining experiments also showed that following vegetative growth, sepX-deficient hyphae did initiate sporulation-specific septation (Supplementary Figure $2 \mathrm{f}-\mathrm{g}$ ). However, sporulation septa that formed under these conditions were irregularly spaced or often appeared tilted, compared to the evenly spaced septa that were visible in wild-type hyphae. To further investigate the importance of SepX for sporulation-specific cell division, we imaged sporulating hyphae of the wildtype and the $\Delta \operatorname{sepX}$ mutant by Transmission Electron Microscopy (TEM) and cryo-Scanning Electron Microscopy (SEM). Inspection of electron micrographs of aerial hyphae revealed that, in contrast to the wildtype and the complemented mutant, little to no sporulation was observed after three days in sepXdeficient hyphae. Given more time (+ 4 days), colonies of the $\Delta s e p X$ mutant did sporulate, but hyphae failed to deposit regularly placed septa, resulting in spores of variable length (Supplementary Figure $2 \mathrm{~h}-\mathrm{j}$ ). In line with the observed spore morphology, we found that spores produced by $\Delta$ sepX mutant were consistently larger and more variable in size compared to spores from the wildtype and the complemented mutant (Figure $2 \mathrm{~d}$-e). Partial septation from one side of the sporogenic hypha was also frequently observed, suggesting FtsZ-mediated constriction had been initiated but then stalled or aborted (Figure 2f). Most strikingly, septation occasionally appeared to occur in an altered plane, often perpendicular to the normal plane of cell division. Taken together our results suggests that SepX plays a crucial role in cell division during vegetative growth and sporulation in Streptomyces.

\section{Lack of hyphal compartmentalization is crucial for fitness}

In addition to the severe cell division defects we observed by electron and light microscopy, colonies formed by the $\Delta s e p X$ mutant also displayed a striking macroscopic phenotype. Wildtype $S$. venezuelae forms roughly circular colonies, that are several millimeters in diameter after three days and produce the green polyketide pigment characteristic of mature $S$. venezuelae spores (Figure $3 a$ ). In contrast, the $\Delta$ sepX mutant grew much more slowly, producing colonies that were small and aberrant in shape (Figure $3 \mathrm{~b}$ ). Colonies remained white, characteristic of a sporulation defect, as has been described for other developmental mutants ${ }^{18}$. This growth phenotype could be complemented by expressing sepX from the native promoter in trans (Figure $3 c$ ). The $\Delta s e p X$ colony morphology is reminiscent of the phenotype of an $\Delta f t s Z$ mutant, which is devoid of cross-walls and sporulation septa (Figure $3 \mathrm{~d})$. Notably, unlike the $f t s Z$ and $\operatorname{sepX}$ deletion mutants, colonies of Streptomyces mutants that produce cross-walls but are specifically blocked in sporulation septation, such as the $\Delta$ whiA mutant ${ }^{14}$ (Figure $3 e$ ), grew to a similar size and morphology as the wildtype. This implies that the inability to synthesize cross-walls and thus compartmentalize the growing mycelium is associated with a severe fitness penalty. 
To further dissect the importance of sepX-dependent cross-wall formation for cellular development and sporulation, we complemented the $\Delta$ sepX mutant with a SepX-mCherry fusion that was specifically expressed during sporulation but not during vegetative growth, thereby impairing cross-wall formation but permitting sporulation-specific cell division. Based on transcriptional profiling data ${ }^{19}$ (Supplementary Figure 3a), we placed sepX-mcherry under the control of the sporulation-specific promoter upstream of the whiE operon, which directs the synthesis of the polyketide that leads to the pigmentation seen in mature Streptomyces spores $^{20}$. Fluorescence microscopy and Western blot analysis confirmed that $P_{\text {whiE }}$ was effective in limiting SepX synthesis to the reproductive growth phase compared to strains in which sepX-mcherry expression was driven either from the constitutive ermE* or the native promoter (Supplementary Figure 3b and c). As expected, the absence of SepX-mCherry during vegetative growth led to an almost complete lack of cross-walls (Supplementary Figure $3 \mathrm{~d}$ ). This conditional sepX mutant was noticeably similar in morphology to the $\Delta$ sepX mutant (Figure 3f). However, sporulation-specific septation occurred readily in this strain to generate spores that were largely regular in size and shape, similar to the $\Delta \operatorname{sepX} / P_{\operatorname{sep} X-\operatorname{sep} X-m c h e r r y}$ complementation strain, suggesting that SepX was functional during sporulation (Supplementary Figure 3e-f). Collectively, these data suggest that SepX-mediated hyphal compartmentalization but not sporulation is crucial for growth and cellular development in Streptomyces.

\section{SepX promotes stable Z-ring formation during sporulation}

Next, to further determine the role of SepX in sporulation-specific cell division, we conducted time-lapse fluorescence microscopy, tracking the assembly of FtsZ-YPet into Z-rings in wildtype $S$. venezuelae and the $\Delta \operatorname{sepX}$ mutant. In the wildtype, following the cessation of hyphal growth, Z-ladder formation occurred rapidly and near synchronously within the tip compartment of a hypha (Supplementary Movie 2). In contrast to the wildtype and in line with the lack of hyphal compartmentalization, we observed frequent cell lysis and highly abundant Z-ladder formation throughout the entire mycelium of the sepX-deficient strain (Figure 4a, b and Supplementary Movie 2 and 3). While the assembly of Z-ladders in the wildtype led to regular septation and the generation of chains of spores, timely completion of the sporulation process in the $\Delta$ sepX mutant appeared much less efficient. This was further confirmed by quantifying the number of spores produced by the wildtype, the $\Delta s e p X$ mutant and the complemented mutant (Supplementary Figure 4c). The visible septation defects in the $\Delta$ sepX mutant at the single-cell level are in agreement with the electron micrographs of $\Delta s e p X$ spore chains, showing the absence of expected septa or incomplete septation (Figure $2 \mathrm{f}$ and Supplementary Figure $2 \mathrm{~h}-\mathrm{i}$ ). Furthermore, kymographs of FtsZ-YPet fluorescence from sporulating wild-type and $\Delta$ sepX hyphae, suggested a clear difference in the dynamics of Zring formation and stability (Figure $4 \mathrm{c}$ and $\mathrm{d}$ ). Analysis of individual Z-rings confirmed the time-dependent and localized increase in FtsZ-YPet fluorescence intensity in wild-type hyphae, indicating the maturation and constriction of Z-rings before they eventually 
disassembled after approximately two hours (Figure 4e). In sepX-deficient hyphae, however, Z-rings assembled much more slowly and displayed a lower average fluorescence intensity. Furthermore, although the spacing of Z-rings was similar to the wildtype (Figure 4f), individual Z-rings in $\Delta$ sepX hyphae were on average spatially less confined compared to wild-type hyphae (Figure 4g). Notably, Western blot analysis confirmed that the alteration in Z-ring dynamics observed in the absence of $\operatorname{sep} X$ was not due to a reduction in the levels of FtsZ. We found that the abundance of FtsZ, the sporulation-specific cell division protein $\mathrm{SsgB}^{8}$ and the developmental regulator $\mathrm{WhiA}^{14}$ was increased compared to the wildtype (Supplementary Figure 4f). This suggests that the absence of $\operatorname{sep} X$, and consequently the lack of cross-walls, affects the abundance of both structural and regulatory proteins involved in cell division, which directly or indirectly influences the correct assembly and architecture of fission-competent Z-rings.

\section{SepX interacts with the cell division machinery}

SepX is comprised of three predicted transmembrane segments connected by two largely disordered and cytoplasmic regions (Figure 1b). Given the lack of any obvious catalytic domain, we reasoned that SepX might fulfil a structural role to support the assembly of the cell division machinery. To test this idea, we performed a targeted bacterial two-hybrid analysis, screening for interaction between SepX and known Streptomyces divisome components, including SepF, the two additional SepF-like proteins SepF2 and SepF3, the two dynamin-like proteins DynA and DynB and the actinomycete-specific cell division protein $\mathrm{SsgB}^{8,11}$. We were unable to test for an interaction with $\mathrm{FtsZ}$ because we previously found that $S$. venezuelae FtsZ is not functional in this assay ${ }^{11}$. Using $\beta$-galactosidase assays to assess the interaction between the individual two-hybrid combinations, we found that SepX could selfinteract and interacted with DynB but did not bind the DynB partner protein DynA or any of the other divisome components tested (Figure 5a and Supplementary Figure 5a). Like SepX, DynB is also associated with the membrane via two transmembrane segments ${ }^{11}$. Our twohybrid results further suggest that the DynB membrane domains may be involved in SepX binding because a DynB mutant version lacking the transmembrane segments failed to bind SepX (Supplementary Figure 5a). To verify that the observed interaction was specific, we also included the transmembrane anti-sigma factor RsbN, which strongly interacts with its cognate sigma factor, $\sigma^{\text {BldN }}\left({ }^{19}\right)$, in our two-hybrid analyses. Importantly, neither SepX nor DynB interacted with RsbN, supporting the notion that SepX specifically associates with DynB (Supplementary Figure 5b).

To further corroborate the two-hybrid results, we performed co-immunoprecipitation coupled to mass spectrometry with a $\triangle$ sepX strain that produced a FLAG-tagged copy of sepX from its native promoter in trans. Sporulating cultures of this strain were cross-linked and SepX-FLAG was recovered from the cell lysate using anti-FLAG antibody-conjugated magnetic beads. Proteins retained on the beads were eluted and analyzed by mass spectrometry. In 
parallel, we performed control experiments with an untagged wild-type strain to identify proteins that bound non-specifically to the anti-FLAG antibody or to the beads. Inspection of obtained peptide profiles revealed a clear enrichment of SepX in the FLAG samples compared to the control samples. Other highly represented proteins in our analysis included metabolic enzymes and transporters. It is conceivable that these proteins are very abundant in the cell and reside in close proximity to SepX, resulting in the observed enrichment of these proteins in our analysis. Importantly, we detected an at least 2-fold enrichment of DynB and its partner protein DynA, and several additional cell division proteins, including SepF, SepF2, SsgB and FtsK (Figure 5b). Previous work had demonstrated that DynB is involved in Z-ring stabilization during sporulation-specific cell division and associates with the divisome by binding to SsgB and SepF2, which in turn are either directly (SsgB) or indirectly (SepF2) connected with the Zring $^{8,11}$. Thus, the enrichment of other key divisome components in our analysis could have been mediated by the SepX-DynB interaction. In summary, our protein-protein interaction studies place SepX in a network of interactions involving key divisome components involved in FtsZ ring positioning, anchoring and stability (Figure 5c).

\section{Sporulation requires SepX or the dynamin-like protein DynB}

We noted that the sporulating hyphae of the $\Delta \operatorname{sep} X$ mutant often displayed asymmetric and incomplete constrictions (Figure $2 \mathrm{f}$ ) This phenotype had previously also been reported for a S. venezuelae strain lacking the two divisome-associated proteins DynA and DynB, which are both required for wildtype-like sporulation ${ }^{11}$. Given that the $\Delta \operatorname{sepX}$ and the $\triangle d y n A B$ mutant share a similar sporulation defect, that all three proteins co-localize with FtsZ at sporulation septa and that SepX and DynB physically interact in vivo, we ask if SepX and DynB are functionally redundant or fulfil an independent role during sporulation-specific cell division.

To address this question, we first tested whether the interaction between SepX and DynB is required for the accumulation of either protein at nascent sporulation septa. Fluorescent microscopy revealed that SepX-mCherry was able to localize to both vegetative cross-walls and sporulation septa in the absence of $d y n A B$ (Figure 6a). Likewise, YPet-labelled DynB accumulated in the expected ladder-like pattern during sporulation in the absence of sepX (Figure 6b). Therefore while, SepX and DynB may interact, this interaction is not required for the recruitment of either component to future division sites. Furthermore, expression of sepX from the constitutive $e^{2} E^{*}$ promoter could not suppress the sporulation defects observed in the $\triangle d y n B$ mutant. Equally, expression of $d y n A B$ from the ermE* promoter could not complement the $\Delta \operatorname{sepX}$ mutant (Supplementary Figure 6a-e, $\mathrm{h}$ and $\mathrm{i}$ ). These findings suggest that DynB and SepX function independently during sporulation-specific cell division.

Since SepX and DynB are both individually important for normal sporulation in $S$. venezuelae, we additionally engineered a strain of $S$. venezuelae that lacked both sepX and $d y n B$. Unlike the parental $\Delta d y n B$ mutant, colonies of the $\Delta \operatorname{sep} X \Delta d y n B$ double mutant were 
small, reflecting the fitness cost we had shown to be associated with the deletion of sepX and the resulting absence of vegetative cross-walls (Figure $6 \mathrm{c}$ ). In contrast to $\Delta d y n B$ and $\Delta s e p X$ single mutants, which sporulate, albeit aberrantly (Supplementary Figure 6a-c), cryo-SEM analysis showed that the $\Delta \operatorname{sep} X \Delta d y n B$ double mutant largely failed to sporulate on solid media and displayed extensive lysis frequently within the mycelium (Figure 6d). Occasionally we observed a very low number of spore-like cells at the outer edge of the colony, which were highly aberrant in size and shape (Supplementary Figure 6f). Complementation of the double mutant with $d y n B$ reconstituted the $\Delta s e p X$ mutant phenotype while complementation with sepX reconstituted the $\Delta d y n B$ mutant phenotype, resulting in irregular sporulation in both cases (Figure 6e). Wildtype-like sporulation could only be restored when the double mutant was complemented with both $\operatorname{sep} X$ and $d y n B$ (Figure 6f).

In addition, we examined the localization of FtsZ-YPet in the $\Delta \operatorname{sep} X \Delta d y n B$ double mutant using time-lapse fluorescence microscopy. During vegetative growth, FtsZ-YPet displayed a similar distribution compared to the $\Delta$ sepX mutant, with FtsZ-ring-like structures that sporadically assembled and continued to migrate in growing hyphae (Supplementary Movie 4). Consistent with the severe growth and septation defects of the $\Delta \operatorname{sep} X \Delta d y n B$ double mutant on solid medium (Figure $6 \mathrm{c}$ ), we also found that the mutant hyphae were prone to frequent large-scale lysis events. In the few hyphae where sporulation septation completed, FtsZ-YPet localization was highly aberrant, with the Z-rings being irregularly laid down and much less spatially confined compared to the Z-rings established in the wildtype (Figure 6g-i and Figure 4c). As observed for the $\Delta \operatorname{sepX}$ mutant strain, FtsZ levels in the $\Delta \operatorname{sep} X \Delta d y n B$ double mutant were similar to those of the wildtype and the $\Delta d y n B$ mutant (Supplementary Figure $6 \mathrm{~g}$ ), indicating the phenotype arises from a defect in the efficient assembly of divisioncompetent Z-rings, rather than insufficient FtsZ.

Finally, given the complexity of the Streptomyces life cycle, we performed phylogenetic analysis to determine whether SepX and DynB are predominantly found in Streptomyces species, or are also present in other actinobacterial systems that divide by binary fission. We first searched 62-representative Streptomycetaceae genomes and confirmed the co-conservation of SepX and DynB homologs throughout the genus (Supplementary Figure 7). In support of the conserved distribution of $\operatorname{sep} X$, we found that expressing the sepX homologue from Streptomyces coelicolor in S. venezuelae can complement the $\Delta$ sepX mutant phenotype and restores normal growth and sporulation (Supplementary Figure 8). This clearly indicates that SepX function is conserved among Streptomyces species. In the wider actinobacteria, a search of 673 representative genomes

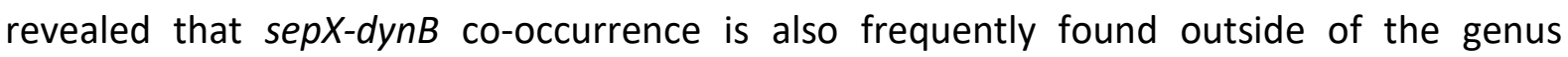
Streptomyces, including in unicellular actinobacteria (Supplementary Figure 9). Although SepX homologs share less than $10 \%$ identical residues, visualization of multiple sequence alignments revealed that residues predicted to reside in the membrane are more conserved (Supplementary Figure 10a and b). These results suggest that the association of SepX with the 
cytoplasmic membrane could be crucial for its function and interaction with DynB during cell division.

Taken together, our results demonstrate that SepX and DynB have distinct but synergistic roles in ensuring the stability and function of the Z-ring in Streptomyces. In addition, sporulation-specific cell division cannot occur effectively in the absence of both SepX and DynB, further underlining their collective importance as central components of the Streptomyces cell division machinery.

\section{DISCUSSION}

The Streptomyces life cycle is characterized by two distinct FtsZ-dependent modes of cell division that lead to the separation of growing hyphal filaments into multigenomic compartments and to the formation and release of unigenomic spores. While some recent progress has been made towards the understanding of the components involved in sporulation-specific sporulation $6,8,11,12$, little has been known about the determinants for Ftszmediated cross-wall formation. Here we describe the previously unknown cell division protein SepX, which is crucial for cross-wall formation and sporulation in Streptomyces. Our results suggest that SepX functions as a structural component of the divisome and contributes to the spatial confinement and stability of the divisome during vegetative growth and sporulation (Figure 7).

Our work reveals that SepX accumulates with FtsZ at future division sites in both vegetative and sporulating hyphae (Figure 1). We note that SepX-mCherry localization appears to be more stable at vegetative septa compared to sporulation septa, implying that in sporulating hyphae SepX localization is more flexible due to the presence of DynB and possibly other sporulation-specific cell division factors. Deletion of sepX causes severe cell division defects during both vegetative and reproductive growth, leading to the lack of hyphal compartmentalization and aberrant sporulation (Figure 2). Importantly, apart from FtsZ, which is essential to form both vegetative and sporulation septa ${ }^{6,21}$, we identified SepX as the first protein specifically required for cross-wall formation.

We show that it is the inability to divide the mycelial network into compartments results in a fitness cost, reduced growth, and aberrant colony morphology (Figure 3). In agreement with previous work using the ftsZ null mutant ${ }^{22}$, we found that in the absence of cross-walls, growing hyphae were prone to lysis. This is in line with the idea that cross-walls protect the mycelial mass from large-scale loss of viability ${ }^{22}$. Furthermore, we propose that the organization of hyphae into compartments could also promote the ordered progression through the Streptomyces life cycle. For example, unlike the wildtype in which Z-rings assemble in the tip compartment of sporogenic hyphae, there is a striking sudden appearance of Z-rings, which spread through the entire mycelium in sepX-deficient hyphae (Figure $4 a$ and b, Supplementary Movie 2 and 3). In line with these results, we found that the levels of FtsZ, 
the Z-ring binding protein SsgB and the transcriptional regulator WhiA were increased in sepXdeficient hyphae compared to the wildtype (Supplementary Figure 4f). However, sporulation appeared to be significantly delayed and less efficient in the absence of $\operatorname{sepX}$ (Supplementary Figure 4c). In support of these results, recent work by Zacharia et al. $(2021)^{23}$ revealed that the spatial organization of specialized cell types within $S$. coelicolor colonies is determined by the ordered spatiotemporal expression of key developmental regulators. Although the exact molecular mechanisms are still unclear, it is conceivable that hyphal compartmentalization during vegetative growth is required for the compartment-specific accumulation of developmentally-regulated proteins or signalling molecules to allow ordered progression through the life cycle.

We further demonstrate that SepX plays a direct role in the assembly process of Z-rings during sporulation. In the wildtype, FtsZ-YPet accumulates as tight, condensed bands that display a time-dependent increase and decrease in fluorescence intensity, which correlates with the assembly, maturation and constriction of Z-rings. However, in sepXdeficient hyphae, Z-rings displayed a lower fluorescence intensity throughout the division process and were on average $10 \%$ wider, indicating that Z-rings are spatially less confined. In support of this, we also note that sporulation septa that formed under these conditions were often placed ectopically and divided spores diagonally or perpendicular to the division plane (Figure $2 \mathrm{f}$ and $2 \mathrm{~g}$ ). A similar observation was recently reported for several point mutants in the early Streptomyces cell division protein $\mathrm{SsgB}^{24}$, suggesting that, like SsgB, SepX plays an important role during the initial stages of divisome formation.

We found that SepX co-purifies with several known Streptomyces divisome components and binds the sporulation-specific divisome component DynB (Figure 5). DynB stabilizes Z-rings via interaction with several divisome components, including DynA, SepF2 and SsgB and likely facilitates the association of SepX with this protein interaction network. Importantly, while SepX and DynB are partially dispensable for sporulation-specific cell division individually, a $\Delta \operatorname{sep} X \Delta d y n B$ double mutant largely fails to produce viable spores (Figure 6). In the rare cases where we did observe a sporulation-like process, individual Z-rings were often loose and displayed a dynamic behavior, including spiral-like FtsZ-structures (Figure 6g), demonstrating the combined importance of SepX and DynB for the spatial stabilization of the divisome and efficient sporulation septation.

How might SepX function in cell division? The sepX gene is not in the vicinity of genes encoding other known divisome components in Streptomyces or the wider actinomycetes (Supplementary Figure 10c). SepX shows no homology to proteins with a known activity but is predicted to be membrane-bound and thus, could act as a membrane anchor for the division machinery. Although we did not detect a direct association between SepX and FtsZ, SepX could mediate its effect on Z-ring assembly and stability via a yet unidentified interaction partner. Further work is required to fully understand the mechanistic role of SepX during cell division and cellular differentiation. 


\section{Methods}

\section{Bacterial strains, oligonucleotides and growth media}

Strains, plasmids and oligonucleotides used in this work are listed in Supplementary Tables 1, 2 and 3 respectively. The Escherichia coli K-12 cloning strains TOP10 or DH5 $\alpha$ were used to propagate plasmids and cosmids. BW $25113^{25}$ containing the $\lambda$ RED plasmid, pIJ790, was used to create disrupted cosmids. When required, the following antibiotics were added to the growth medium: $100 \mu \mathrm{g} / \mathrm{ml}$ carbenicillin $50 \mu \mathrm{g} / \mathrm{ml}$ kanamycin, $25 \mu \mathrm{g} / \mathrm{ml}$ hygromycin, $50 \mu \mathrm{g} / \mathrm{ml}$ apramycin, or $25 \mu \mathrm{g} / \mathrm{ml}$ chloramphenicol.

S. venezuelae was cultured in maltose-yeast extract-malt extract medium (MYM), supplemented with R2 trace element solution at 1:500. Liquid cultures were grown in flasks with springs at $30{ }^{\circ} \mathrm{C}$ at $250 \mathrm{rpm}$. When required, MYM agar contained $5 \mu \mathrm{g} / \mathrm{ml}$ kanamycin, $25 \mu \mathrm{g} / \mathrm{ml}$ hygromycin, $50 \mu \mathrm{g} / \mathrm{ml}$ apramycin or $50 \mu \mathrm{g} / \mathrm{ml}$ thiostrepton. Cosmids and plasmids were conjugated from the E. coli strain ET12567 containing pUZ8002 ${ }^{26}$ into $S$. venezuelae as described previously by Bush et al. ${ }^{14}$.

\section{Construction and complementation of the $\Delta \operatorname{sepX}$ mutant}

Using 'Redirect' PCR targeting ${ }^{27}$, the $\Delta s e p X$ mutant was generated in which the central (968 bp) coding region was replaced with a single apramycin resistance cassette. Cosmid 5E03 (http://strepdb.streptomyces.org.uk/) was introduced into E. coli BW25113 containing plJ790 and the sepX gene ( $v n z$ 14865) was replaced with the apr-oriT cassette amplified from pIJ773 using the primer pairs mb266 and mb267. The resulting disrupted cosmids were confirmed by PCR analysis using the flanking primers mb1431 and mb1432, and introduced into $S$. venezuelae by conjugation via E. coli ET12567/pUZ8002. Homologous recombination gave rise to two distinct morphotypes of exconjugants and their genotypes were confirmed using the flanking primers mb1431 and mb1432. The faster-growing and larger exconjugants were all confirmed to be single cross-over $\left(\mathrm{Apr}^{\mathrm{R}}, \mathrm{Kan}^{\mathrm{R}}\right)$ strains in which the wild-type gene was still present. All double cross-over strains $\left(\mathrm{Apr}^{\mathrm{R}}, \mathrm{Kan}^{\mathrm{S}}\right)$ arose from smaller exconjugants that took 7-10 days to emerge. A representative sepX null mutant was designated SV55. For complementation, pMB182 was introduced into the $\Delta s e p X$ mutant by conjugation.

\section{Construction and complementation of the $\Delta \operatorname{sep} X \Delta d y n B$ double mutant}

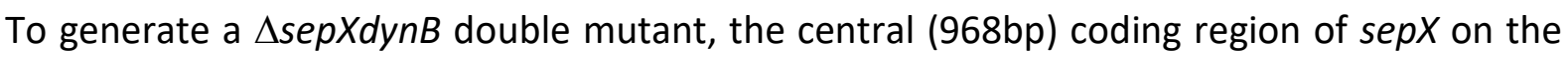
5 E03 cosmid was instead replaced with the hyg-oriT cassette amplified from plJ10700 using the identical primer pair mb266 and mb267. Disrupted cosmids were confirmed by PCR using mb1431 and mb1432 and introduced into the S. venezuelae dynB::apr mutant (SS2) by conjugation. As for the $\Delta$ sepX::apr mutant (SV55), double cross-overs originated from slowgrowing exconjugants $\left(\mathrm{Hyg}^{\mathrm{R}}, \mathrm{Kan}^{\mathrm{S}}\right)$ and were confirmed via PCR-analysis using mb1431 and mb1432. A representative $\Delta$ sepX::hyg $\triangle d y n B:: a p r$ null mutant was designated SV57. For complementation, plJ10257 and the pIJ10257-derived plasmids pSS64 and pMB156 were 
introduced into the SV57 mutant by conjugation, serving to complement in trans either sepX, $d y n B$ or both sepX and $d y n B$.

\section{Analysis of spore morphology and sporulation efficiency}

For analysis of spore size distributions, triplicate spore preparations were made in $3 \mathrm{ml}$ of $20 \%$ glycerol and suspended in Isoton II (Beckman Coulter, Brea, CA, USA). The particle volume was then measured for a minimum of 100000 particles per sample using a Multisizer $4 \mathrm{e}$ Coulter counter (Beckman Coulter) equipped with a $30 \mu \mathrm{m}$ aperture tube (size range $0.6 \mu \mathrm{m}$ to $18 \mu \mathrm{m}$, aperture current $600 \mu \mathrm{A}$ ). All measurements were conducted with logarithmic spacing into 400 bins using the Multiszier 4 software (Version 4.01) but are presented on a linear $\mathrm{x}$-axis for clarity. For analysis of spore efficiency, triplicate spore preparations were measured in the same way but instead counting the total number of particles in $50 \mu$ l. The dilution factor (1:500 for all samples) was then used to calculate the number of particles per $\mu \mathrm{l}$ as presented.

\section{Cryo-scanning electron microscopy}

Streptomyces samples were mounted on an aluminium stub using Tissue Tek $^{R}$ (BDH Laboratory Supplies, Poole, England) and plunged into liquid nitrogen slush. The sample was transferred onto the cryo-stage of an ALTO 2500 cryo-transfer system (Gatan, Oxford, England) attached to either a Zeiss Supra 55 VP FEG scanning electron microscope (Zeiss SMT, Germany) or an FEI Nova NanoSEM 450 (FEI, Eindhoven, The Netherlands). Sublimation of surface frost was performed at $-95^{\circ} \mathrm{C}$ for three minutes before sputter coating with platinum for $150 \mathrm{~s}$ at $10 \mathrm{~mA}$. The sample was moved onto the main cryo-stage in the microscope for imaging at $3 \mathrm{kV}$ and digital TIFF files were stored. All uncropped SEM images, including additional images of the same strain, are shown in Supplementary figure 11-15.

\section{Transmission electron microscopy}

Streptomyces colonies were excised from agar plates and fixed overnight in $2.5 \%(\mathrm{v} / \mathrm{v})$ glutaraldehyde in $0.05 \mathrm{M}$ sodium cacodylate, $\mathrm{pH} 7.3$ and post-fixed in $1 \%(\mathrm{w} / \mathrm{v}) 0 \mathrm{OSO}$ in $0.05 \mathrm{M}$ sodium cacodylate for $1 \mathrm{~h}$ at room temperature. After three, 15-min washes in distilled water, they were dehydrated through an ethanol series and infiltrated with LR White resin (London Resin Company, Reading, Berkshire) which was polymerized at $60^{\circ} \mathrm{C}$ for $16 \mathrm{hrs}$. Using a Leica UC6 ultramicrotome (Leica, Milton Keynes), 90nm sections were cut and stained with $2 \%(\mathrm{w} / \mathrm{v})$ uranyl acetate and $1 \%(\mathrm{w} / \mathrm{v})$ lead citrate before viewing at either $80 \mathrm{kV}$ in a Jeol 1200 transmission electron microscope (Jeol UK, Welwyn Garden City) or at 200 kV in an FEI Tecnai 20 transmission electron microscope (FEI UK Ltd, Cambridge) and imaged using an AMT digital camera (Deben, Bury St Edmunds, UK) to record TIFF files.

\section{Light microscopy and image analysis}

For imaging protein localization in S. venezuelae, cells were grown in MYM medium for 10$20 \mathrm{~h}$ and a $2-\mu \mathrm{L}$ sample of the culture was immobilized on a $1 \%$ agarose pad. Streptomyces 
hyphae or spores were visualized using a Zeiss Axio Observer Z.1 inverted epifluorescence microscope fitted with a sCMOS camera (Hamamatsu Orca FLASH 4), a Zeiss Colibri 7 LED light source, a Hamamatsu Orca Flash 4.0v3 sCMOS camera, and a temperature-controlled incubation chamber. Images were acquired using a Zeiss Plan-Apochromat 100x/NA 1.4 Ph3 objective or a Zeiss Alpha Plan-Apo 100x/1.46 Oil DIC M27 objective with the following excitation/emission bandwidths: YFP (489-512nm/520-550nm), mCherry (577-603nm/614659nm), CFP (401-445nm/460-479nm) and FM4-64 (577-603nm/614-759nm). Still images and time-lapse images series were collected using Zen Blue (Zeiss) and analyzed using Fiji ${ }^{28}$.

Time-lapse fluorescence imaging of FtsZ-YPet and SepX-mCherry localization in S. venezuelae was performed as previously described ${ }^{29}$. Briefly, S. venezuelae strains were grown in MYM medium for about $36 \mathrm{~h}$ at $30^{\circ} \mathrm{C}$ and $250 \mathrm{rpm}$ to reach complete sporulation. To isolate spores, mycelium was pelleted at $400 \times g$ for $1 \mathrm{~min}$. Spores were loaded into B04A microfluidic plates (ONIX, CellASIC), allowed to germinate and grown by perfusing MYM for 3 $\mathrm{h}$ before medium was switched to spent-MYM medium. Spent-MYM was prepared from the 36-hour sporulation culture by filtering the growth medium to remove spores and mycelia fragments using a $0.22 \mu \mathrm{m}$ syringe filter. The media flow rate and temperature were maintained at $2 \mathrm{psi}$ and $30^{\circ} \mathrm{C}$. Time-lapse imaging was started approximately $8 \mathrm{~h}$ after spores had germinated and images were acquired every 10-20 min, respectively, until sporulation was completed. For the detection of SepX-mCherry, imaging intervals were increased to 40 $\min$.

For co-localization analysis, frames that showed a clear SepX-mCherry and FtsZ-YPet signal in sporulating hyphae were identified. Images were corrected for background fluorescence using the "subtracted_measured_background" plugin in Fiji ${ }^{30}$ and the resulting fluorescence intensities for the selected hyphae were visualized using GraphPad Prism 9.

Kymograph and subsequent image analysis was performed as described in RamosLéon et al. (2021) ${ }^{12}$. Briefly, kymographs were generated from registered time-lapse image series of strain SS12 (WT/ftsZ-ypet) and SV55 (AsepX/ftsZ-ypet) using Fiji Reslice plugin. Hyphae undergoing sporulation septation were first identified based on the characteristic FtsZ-YPet localization pattern following the cessation of tip extension. 24 frames (10 $\mathrm{min}$ (frame) including one frame immediately before and 22 frames after the cessation of hyphal growth were isolated. Selected hyphae were "straightened" and a segmented line (5 pt) was manually drawn along the center of the straightened hyphae. FtsZ-YPet fluorescence intensity was plotted along this line as a function of time (240 $\mathrm{min}$ ) using the "Reslice" command. Kymographs were further annotated in Adobe Illustrator CS6. To visualize fluorescence intensities of Z-rings over time, time-lapse series were first corrected for background fluorescence by applying a custom Python script with a multi-Otsu thresholding algorithm ${ }^{12}$. The following steps were performed in Fiji: Z-rings were identified manually in time-lapse series and an ROI of 10x20 pixels was drawn around each Z-ring. The average fluorescence intensity values within each ROI were then collected and the mean fluorescence intensity trace of all Z-rings isolated from either wild-type or sepH-deficient hyphae was plotted using Graphpad Prism 9. 
To determine the width and distance of Z-rings, an average fluorescence intensity projection for each of the time-series was first generated in Fiji. The corresponding fluorescence intensity trace along a segmented line (5 pt) manually drawn along the hyphal midline was then extracted, and the obtained data was further processed in R. For each strain, five independent time-lapse series were analysed. Peaks (which correspond to potential Zrings) were identified using a custom $\mathrm{R}$ script and further filtered to remove false-positive peaks with a fluorescence intensity below 100 . Z-ring width was calculated by measuring the full width at half maximum of the Z-ring peak in the fluorescence intensity profiles and Z-ring spacing was determined by measuring the distance between neighbouring peaks. Z-ring width and distance values were plotted using Graphpad Prism 9.

\section{FM 4-64 staining}

For membrane staining, Streptomyces hyphae were incubated with $0.5 \mathrm{mg} / \mathrm{ml} \mathrm{FM} \mathrm{4-64} \mathrm{Dye}$ (N-(3-Triethylammoniumpropyl)24-(6-(4-(Diethylamino) Phenyl) Hexatrienyl) Pyridinium Dibromide) (Molecular Probes) for $15 \mathrm{~min}$ in the dark. Hyphae were then directly spotted onto a $1 \%$ agarose pad and visualized using a Zeiss Axio Observer Z1 microscope using an Alpha Plan-Apo $100 \times / 1.46$ Oil DIC M27 objective. Spores of SV55 were loaded into a BA04 microfluidic plate (ONIX, CellASIC) and allowed to germinate and to grow using constant

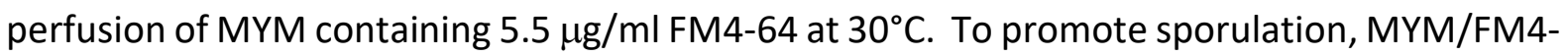
64 medium was replaced after 3 h of growth by "spent-MYM"/FM4-64. The "spent-MYM" was prepared as described previously ${ }^{34}$. Hyphae were visualized using a Zeiss Axio Observer Z1 microscope equipped with a Plan Apochromat 100x/1.4 Oil Ph3 objective. Images were collected and analyzed using ZenBlue (Zeiss) or Fiji ${ }^{28}$. We note that FM4-64 staining using the ONIX microfluidics systems is inefficient as the membrane dye appears to bind to the internal plate material. Thus, the majority of images shown in the manuscript were generated using cells immobilized on agarose pads.

\section{Cross-wall staining and analysis}

For HADA (7-hydroxycoumarin 3-carboxylic acid-amino-D-alanine) labelling ${ }^{16}$, spores of the wild type carrying an empty plasmid (SS4), SV55, MB182, SS5, SS414 or MB1120 were loaded into BA04 microfluidic plates (ONIX, CellASIC). Trapped spores were continuously supplied with MYM containing 0.25 or $0.5 \mathrm{mM} \mathrm{HADA}$ at $2 \mathrm{psi}$ at $30^{\circ} \mathrm{C}$. Following spore germination, hyphae were allowed to grow by perfusing MYM-HADA at 2 psi for 4-5 h. Prior to image acquisition MYM-HADA was replaced with MYM and hyphae were visualized using a Zeiss Axio Observer Z1 microscope fitted with a 63x/1.4 Oil BF/DICIII objective. Images were collected using Zen Blue (Zeiss) and analyzed with $\mathrm{Fiji}^{28}$. To determine the number of cross-walls per hyphae, the length of individual hyphae originating from single spores was measured manually and the number of cross-walls present in the respective hypha was recorded. Hyphae from at least 20 independent spores were analyzed per replicate $(n=3)$. Distribution of cross-walls per strain and hyphal length was plotted using GraphPad Prism 9. 


\section{Automated Western blotting}

Samples of frozen mycelium, originating from 2-5 ml liquid MYM cultures, were resuspended in $0.4 \mathrm{ml}$ ice-cold sonication buffer [ $20 \mathrm{mM}$ Tris pH 8.0, $5 \mathrm{mM}$ EDTA, 1x EDTA-free protease inhibitors (Sigma Aldrich)] and sonicated (5x $15 \mathrm{sec}$ on/15 sec off) at 4.5-micron amplitude. Lysates were then centrifuged at $16,000 \times \mathrm{g}$ for $15 \mathrm{~min}$ at $4^{\circ} \mathrm{C}$ to remove cell debris. Total protein concentration was determined using the Bradford assay (Biorad). For anti-FtsZ experiments, $1 \mu \mathrm{g}$ of total protein from each time point was loaded in triplicate into a microplate (ProteinSimple \#043-165) and anti-FtsZ antibody (Polyclonal, Cambridge Research Biochemicals $)^{12}$ diluted 1:200. FtsZ levels, originating from the wildtype (WT), WT/ftsZ-ypet (SS12), $\Delta$ sepX (SV55), $\Delta$ sepX/ftsZ-ypet (MB180), $\Delta d y n B$ (SS2), $\Delta \operatorname{sepX\Delta dynB~(SV57),~}$

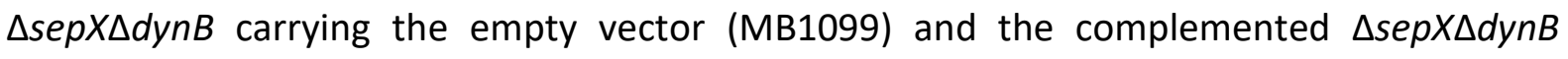
(MB1103) strains were then assayed using the automated Western blotting machine WES (ProteinSimple, San Jose, CA), according to the manufacturer's guidelines. The $\Delta$ ftsZ (DU669) strain was used as a negative control. For the detection of mCherry protein fusions, $2.5 \mu \mathrm{g}$ of total protein and anti-mCherry (Abcam 183628) at a 1:200 dilution was used. mCherry levels, originating from wild-type, $\triangle f t s Z$ and $\triangle d y n A B$ strains (as negative controls) or additionally

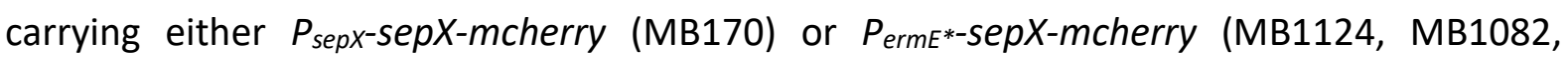
MB1092) were then assayed in the same way. For the detection of YPet protein fusions, $1 \mu \mathrm{g}$ of total protein and anti-GFP (Sigma SAB4301138) at 1:500 dilution was used. For detection of SsgB in WT/ftsZ-ypet (SS12) and $\Delta$ sepX/ftsZ-ypet (MB180), $2.5 \mu \mathrm{g}$ of total protein and antiSsgB (Polyclonal, Cambridge Research Biochemicals) at 1:100 dilution was used. For detection of WhiA in WT/ftsZ-ypet (SS12) and $\Delta$ sepX/ftsZ-ypet (MB180), $0.5 \mu \mathrm{g}$ of total protein and antiWhiA $^{14}$ (Polyclonal, Cambridge Research Biochemicals) at 1:100 dilution was used. Virtual Western blots were generated using the Compass software for simple western (Version 6.0.0) and uncropped images of all blots are shown in Supplementary Figure 16.

\section{SsgB antibody production}

The gene encoding SsgB, vnz_05545, was cloned into pTB146, generating pSS440. Heterologous protein production was induced with $1 \mathrm{mM} \mathrm{IPTG}$ at $30^{\circ} \mathrm{C}$ for $4 \mathrm{~h}$. Cells were harvested by centrifugation and resuspended in $50 \mathrm{mM}$ Tris- $\mathrm{HCl} \mathrm{pH} \mathrm{8.0,1} \mathrm{M} \mathrm{NaCl}, 5 \%$ glycerol, 1\% Triton-X. His 6 -SUMO-SsgB was purified using an HisTrap column (GE Healthcare) and eluted with an increasing imidazole concentration. The protein was dialysed overnight at $4{ }^{\circ} \mathrm{C}$ in buffer containing $50 \mathrm{mM}$ Tris- $\mathrm{HCl}$ pH 8.0, $150 \mathrm{mM} \mathrm{NaCl}, 10 \%$ glycerol, $1 \mathrm{mM}$ DDT and His ${ }_{6}^{-}$ Upl1 protease at a 1:100 ratio. The released $\mathrm{His}_{6}$-SUMO tag and $\mathrm{His}_{6}-\mathrm{Upl}$ 1 protease were removed by incubation with Ni-NTA affinity agarose beads and a total amount of $2 \mathrm{mg} \mathrm{ml}^{-1}$ of untagged SsgB was sent to Cambridge Research Biochemicals (UK) to raise antibodies in rabbits. 


\section{Immunoprecipitation for SepX-mCherry detection}

To be able to detect SepX-mCherry in whole cell lysates, expressed from either the native sepX promoter or the whiE promoter, immunoprecipitation was first conducted using RFPTrap agarose (Chromotek) prior to automated Western blot analysis. The $\Delta$ sepX (SV55), WT/PermE*-sepX-mcherry (MB1124), $\Delta$ sepX/Psepx-sepX-mcherry (MB171) and $\Delta$ sepX/P $P_{\text {wiE- }}$ sepX-mcherry (MB1120) strains were grown in $30 \mathrm{ml} M Y M$ liquid cultures at $30^{\circ} \mathrm{C}$ and 250 rpm to the required growth stage as confirmed by microscopy. Two cultures for each strain were pooled, pelleted, and washed twice with PBS before resuspension in $1.5 \mathrm{ml}$ lysis buffer (10 mM Tris-Cl pH 7.5, 150 mM NaCl, 0.5 mM EDTA, 1\% Triton-X-100, 10 mg/ml lysozyme, $1 \mathrm{x}$ EDTA-free protease inhibitors [Sigma Aldrich]). Samples were incubated for $25 \mathrm{~min}$ at $37^{\circ} \mathrm{C}$, placed on ice and lysed by sonication using $8 \times 20 \mathrm{sec}$ at 8 microns with $1 \mathrm{~min}$ on ice between each cycle. Lysates were then centrifuged twice at $4^{\circ} \mathrm{C}$ and $16,000 \times g$ and the supernatant retained. Total protein concentration was determined by Bradford assay (Biorad) and the lysates were normalized to ensure equal protein input. $25 \mu \mathrm{l}$ of binding control agarose beads (Chromotek; bab20), prepared in dilution buffer (10 mM Tris-Cl pH 7.5; $150 \mathrm{mM} \mathrm{NaCl}, 0.5 \mathrm{mM}$ EDTA) were added to each lysate and left to rotate end-over-end for $1 \mathrm{~h}$ at $4^{\circ} \mathrm{C}$. The beads were sedimented at $2,500 \times g$ and $4^{\circ} \mathrm{C}$ for $5 \mathrm{~min}$ and the supernatant was retained. RFP-Trap agarose beads (Chromotek; rta20) were then added and the lysates again left to rotate endover-end for $1 \mathrm{~h}$ at $4^{\circ} \mathrm{C}$. The beads were washed with ice-cold lysis buffer according to the manufacturer's instructions and eluted in $50 \mu \mathrm{l} 2 \mathrm{x}$ SDS dye. $0.5 \mu \mathrm{l}$ of each sample was subsequently loaded into a microplate for automated Western blot analysis and mCherry levels were determined as described above.

\section{Co-immunoprecipitation and mass spectrometry}

Co-immunoprecipitation experiments using SepX as bait were performed using the SepXFLAG/DynB-YPet stain (MB942) and the DynB-YPet strain (SS92) as a negative control. Duplicate $30 \mathrm{ml} \mathrm{MYM} \mathrm{liquid} \mathrm{cultures} \mathrm{were} \mathrm{grown} \mathrm{at} 30^{\circ} \mathrm{C}$ and $250 \mathrm{rpm}$ until the sporulation growth stage, as judged by the detection of DynB-YPet, localized to spore septa using fluorescence microscopy. Cultures were pooled, and formaldehyde (Sigma F8775) added to a final concentration of $1 \%$. Cultures were further incubated at $30^{\circ} \mathrm{C}$ and $250 \mathrm{rpm}$ for $30 \mathrm{mins}$ before quenching the reaction by adding glycine to a final concentration of $125 \mathrm{mM}$. Cells were pelleted at $4^{\circ} \mathrm{C}$ and washed twice with ice-cold PBS, resuspended in $1.5 \mathrm{ml}$ lysis buffer (10 mM Tris-Cl pH 8, $150 \mathrm{mM} \mathrm{NaCl}, 1 \%$ Triton-X-100, $10 \mathrm{mg} / \mathrm{ml}$ lysozyme, 1x EDTA-free protease inhibitors [Sigma Aldrich]) and incubated for $25 \mathrm{~min}$ at $37^{\circ} \mathrm{C}$. Samples were then lysed by sonication at $8 \times 20 \mathrm{sec}$ at 8 microns with $1 \mathrm{~min}$ on ice between each cycle before being centrifuged twice at $4^{\circ} \mathrm{C}$ and $16,000 \times g$, retaining the supernatant. SepX-FLAG was pulled down using the $\mu$ MACS epitope tag protein isolation kit (FLAG 130-101-591) and eluted using Triethylamine $\mathrm{pH} 11.8$ as described in the manufacturer's instructions. Equal volumes of $2 x$ SDS-dye were added to the eluates before heating at $95^{\circ} \mathrm{C}$ for $3 \mathrm{~min}$. Equal volumes were then loaded onto a $10 \%$ acrylamide gel, ran briefly and each sample was cut out of the 
gel. Proteins contained within the gel slices were digested with trypsin for $8 \mathrm{hrs}$ at pH 7.5 and $40^{\circ} \mathrm{C}$ and prepared for liquid chromatography-tandem mass spectrometry (LC-MS/MS) as described previously ${ }^{31}$ using an Orbitrap Eclipse tribrid mass spectrometer (Thermo Fisher Scientific) and a nanoflow high-performance liquid chromatography (HPLC) system (Dionex Ultimate3000, Thermo Fisher Scientific). MS/MS peaks were analyzed using Mascot v.2.3 (Matrix Science. All Mascot searches were collated and verified with Scaffold (Proteome Software Version 4.0.7). Accepted proteins passed the following threshold in Scaffold: $95 \%$ confidence for protein match and minimum of two unique peptide matches with $95 \%$ confidence.

For analysis in R (version 3.6.0) ${ }^{32}$, the "Quantitative values" in Scaffold from both the MB942 and SS92 strains, generated from each of three independent experiments were brought together into one dataframe. The function impute.knn (parameters $k=3$ and rowmax $=0.9$ ) (package impute) was used to impute the missing values. The values were made into a DGEList object using the function DGEList (package edgeR). Appropriate design and contrast matrices were made using the functions model.matrix and makeContrasts (package limma). Function calcNormFactors (package edgeR) was used to calculate normalisation factors and the voom function (package limma) to log-transform the data and calculate appropriate observation level weights. To fit linear models the ImFit function was applied followed by contrasts.fit to compute coefficients (package limma). Differential expression values were calculated using the eBayes function (package limma). The complete data set is available in the Source Data.

\section{Bacterial adenylate cyclase two-hybrid assays}

To test the interaction between full-length or truncated proteins, coding sequences were amplified using the primers listed in Supplementary Table 1 and cloned in-frame to create the vectors listed in the same table. E. coli BTH101 was then co-transformed with 'T25' and 'T18' fusion plasmids. $\beta$-galactosidase activity was assayed in biological triplicate in 96-well plates $^{33}$.

\section{Actinobacterial phylogeny and SepX/DynB ortholog identification}

A search set of 673 Actinobacterial genomes were chosen based on annotation as 'reference' or 'representative' at GenBank as of November 2017, along with 10 genomes from Phylum Chloroflexi that serve as outgroups. The Chloroflexi genome Anaerolinea thermophila UNI-1 was used to root the tree. Amino acid sequences of 37 conserved housekeeping genes were automatically identified, aligned, and concatenated using Phylosift ${ }^{34}$. Model selection was performed using SMS ${ }^{35}$ implemented at http://www.atgc-montpellier.fr/phyml/ ${ }^{36}$, which resulted in selection of a LG substitution model with gamma distributed rate variation between sites. Phylogenetic reconstruction was performed by RAxML version 8.2.10 ${ }^{37}$ with 100 rapid bootstraps replicates to assess node support. The tree was visualized and formatted using $\mathrm{iTOL}^{38}$. Taxonomic assignments were based on the taxonomy database maintained by NCBI (https://www.ncbi.nIm.nih.gov/Taxonomy/Browser/wwwtax.cgi). SepX and DynB 
orthologs were identified based on a reciprocal BLAST search (e-value cutoff $=1 \mathrm{e}-6$ ) of the same set of 673 Actinobacterial genomes, using the $S$. venezuelae SepX/DynB sequences as a query ( $v n z$ _14865 and vnz_12105, respectively). For members of the genus Streptomyces, this ortholog search was additionally performed on available genomes associated with each species' NCBI Taxonomy ID as of October 2020.

Multiple sequence alignments of SepX homologs from Streptomyces and the wider actinobacteria were visualized using Jalview $^{39}$ and gene neighborhood analysis was performed using the webFlaGs server ${ }^{40}$.

\section{Data availability statement}

Additional data is available on request from the corresponding author. Source Data are provided with this paper. 


\section{References}

1. Wildermuth, H. \& Hopwood, D. A. Septation during sporulation in Streptomyces coelicolor. J Gen Microbiol 60, 51-59 (1970).

2. Becher, P. G. et al. Developmentally regulated volatiles geosmin and 2-methylisoborneol attract a soil arthropod to Streptomyces bacteria promoting spore dispersal. Nature Microbiology 5, 821-829 (2020).

3. Bobek, J., Šmídová, K. \& Čihák, M. A Waking Review: Old and Novel Insights into the Spore Germination in Streptomyces. Front. Microbiol. 8, 2205 (2017).

4. Bisson-Filho, A. W. et al. Treadmilling by FtsZ filaments drives peptidoglycan synthesis and bacterial cell division. Science 355, 739-743 (2017).

5. Yang, X. et al. GTPase activity-coupled treadmilling of the bacterial tubulin FtsZ organizes septal cell wall synthesis. Science 355, 744-747 (2017).

6. Cantlay, S., Sen, B. C., Flärdh, K. \& McCormick, J. R. Influence of core divisome proteins on cell division in Streptomyces venezuelae ATCC 10712. Microbiology (2021) doi:10.1099/mic.0.001015.

7. McCormick, J. R. Cell division is dispensable but not irrelevant in Streptomyces. Curr. Opin. Microbiol. 12, 689-698 (2009).

8. Willemse, J., Borst, J. W., Waal, E. de, Bisseling, T. \& Wezel, G. P. van. Positive control of cell division: FtsZ is recruited by SsgB during sporulation of Streptomyces. Genes Dev. 25, 89-99 (2011).

9. Duman, R. et al. Structural and genetic analyses reveal the protein SepF as a new membrane anchor for the $Z$ ring. PNAS 110, E4601-E4610 (2013).

10. Gola, S., Munder, T., Casonato, S., Manganelli, R. \& Vicente, M. The essential role of SepF in mycobacterial division. Molecular Microbiology 97, 560-576 (2015).

11. Schlimpert, S. et al. Two dynamin-like proteins stabilize FtsZ rings during Streptomyces sporulation. PNAS 114, E6176-E6183 (2017).

12. Ramos-León, F. et al. A conserved cell division protein directly regulates FtsZ dynamics in filamentous and unicellular actinobacteria. eLife 10, e63387 (2021).

13. Mistry, B. V., Sol, R. D., Wright, C., Findlay, K. \& Dyson, P. FtsW Is a Dispensable Cell Division Protein Required for Z-Ring Stabilization during Sporulation Septation in Streptomyces coelicolor. Journal of Bacteriology 190, 5555-5566 (2008).

14. Bush, M. J., Bibb, M. J., Chandra, G., Findlay, K. C. \& Buttner, M. J. Genes Required for Aerial Growth, Cell Division, and Chromosome Segregation Are Targets of WhiA before Sporulation in Streptomyces venezuelae. mBio 4, e00684-13 (2013).

15. Bush, M. J., Chandra, G., Bibb, M. J., Findlay, K. C. \& Buttner, M. J. Genome-Wide Chromatin Immunoprecipitation Sequencing Analysis Shows that WhiB Is a Transcription Factor That Cocontrols Its Regulon with WhiA To Initiate Developmental Cell Division in Streptomyces. mBio 7, e00523-16, /mbio/7/2/e00523-16.atom (2016).

16. Kuru, E., Tekkam, S., Hall, E., Brun, Y. V. \& Van Nieuwenhze, M. S. Synthesis of fluorescent $\mathrm{D}$-amino acids and their use for probing peptidoglycan synthesis and bacterial growth in situ. Nat Protoc 10, 33-52 (2015). 
17. Bibb, M. J., Janssen, G. R. \& Ward, J. M. Cloning and analysis of the promoter region of the erythromycin-resistance gene (ermE) of Streptomyces erythraeus. Gene 41, E357E368 (1986).

18. Ryding, N. J. et al. New Sporulation Loci in Streptomyces coelicolor A3(2). Journal of Bacteriology 181, 5419-5425 (1999).

19. Bibb, M. J., Domonkos, Á., Chandra, G. \& Buttner, M. J. Expression of the chaplin and rodlin hydrophobic sheath proteins in Streptomyces venezuelae is controlled by oBldN and a cognate anti-sigma factor, RsbN. Molecular Microbiology 84, 1033-1049 (2012).

20. Kelemen, G. H. et al. Developmental Regulation of Transcription of whiE, a Locus Specifying the Polyketide Spore Pigment in Streptomyces coelicolor A3(2). J Bacteriol 180, 2515-2521 (1998).

21. McCormick, J. R., Su, E. P., Driks, A. \& Losick, R. Growth and viability of Streptomyces coelicolor mutant for the cell division gene ftsZ. Mol. Microbiol. 14, 243-254 (1994).

22. Santos-Beneit, F., Roberts, D. M., Cantlay, S., McCormick, J. R. \& Errington, J. A mechanism for FtsZ-independent proliferation in Streptomyces. Nat Commun 8, 1378 (2017).

23. Zacharia, V. M. et al. Genetic Network Architecture and Environmental Cues Drive Spatial Organization of Phenotypic Division of Labor in Streptomyces coelicolor. mBio 12, (2021).

24. Xiao, X. et al. Ectopic positioning of the cell division plane is associated with single amino acid substitutions in the FtsZ-recruiting SsgB in Streptomyces. Open Biology 11, 200409.

25. Paget, M. S. B., Chamberlin, L., Atrih, A., Foster, S. J. \& Buttner, M. J. Evidence that the Extracytoplasmic Function Sigma Factor $\zeta$ E Is Required for Normal Cell Wall Structure in Streptomyces coelicolor A3(2). Journal of Bacteriology 181, 204-211 (1999).

26. Gust, B., Challis, G. L., Fowler, K., Kieser, T. \& Chater, K. F. PCR-targeted Streptomyces gene replacement identifies a protein domain needed for biosynthesis of the sesquiterpene soil odor geosmin. PNAS 100, 1541-1546 (2003).

27. Gust, B. et al. $\lambda$ Red-Mediated Genetic Manipulation of Antibiotic-Producing Streptomyces - ScienceDirect. Advances in Applied Microbiology 54, 107-128 (2004).

28. Schindelin, J. et al. Fiji - an Open Source platform for biological image analysis. Nat Methods 9, (2012).

29. Schlimpert, S., Flärdh, K. \& Buttner, M. Fluorescence Time-lapse Imaging of the Complete S. venezuelae Life Cycle Using a Microfluidic Device. J Vis Exp (2016) doi:10.3791/53863.

30. Goedhart, J. PlotTwist - a web app for plotting and annotating time-series data. 745612 https://www.biorxiv.org/content/10.1101/745612v2 (2019) doi:10.1101/745612.

31. Bender, K. W. et al. Autophosphorylation-based Calcium ( $\mathrm{Ca} 2+)$ Sensitivity Priming and $\mathrm{Ca} 2+/$ Calmodulin Inhibition of Arabidopsis thaliana Ca2+-dependent Protein Kinase 28 (CPK28). J Biol Chem 292, 3988-4002 (2017).

32. R Core Team (2019) R: A Language and Environment for Statistical Computing. R Foundation for Statistical Computing, Vienna, Austria. - Google Search. 
https://www.google.com/search?client=firefox-b-

$\mathrm{d} \& \mathrm{q}=\mathrm{R}+$ Core + Team $+\% 282019 \% 29+\mathrm{R} \% 3 \mathrm{~A}+\mathrm{A}+$ Language+and+Environment+for + Statistica I+Computing.+R+Foundation+for+Statistical+Computing\%2C+Vienna\%2C+Austria.

33. Griffith, K. L. \& Wolf, R. E. Measuring $\beta$-Galactosidase Activity in Bacteria: Cell Growth, Permeabilization, and Enzyme Assays in 96-Well Arrays. Biochemical and Biophysical Research Communications 290, 397-402 (2002).

34. Darling, A. E. et al. PhyloSift: phylogenetic analysis of genomes and metagenomes. PeerJ 2, e243 (2014).

35. Lefort, V., Longueville, J.-E. \& Gascuel, O. SMS: Smart Model Selection in PhyML. Mol. Biol. Evol. 34, 2422-2424 (2017).

36. Guindon, S. et al. New Algorithms and Methods to Estimate Maximum-Likelihood Phylogenies: Assessing the Performance of PhyML 3.0. Syst Biol 59, 307-321 (2010).

37. Stamatakis, A. RAxML version 8: a tool for phylogenetic analysis and post-analysis of large phylogenies. Bioinformatics 30, 1312-1313 (2014).

38. Letunic, I. \& Bork, P. Interactive Tree Of Life (iTOL) v4: recent updates and new developments. Nucleic Acids Res. 47, W256-W259 (2019).

39. Waterhouse, A. M., Procter, J. B., Martin, D. M. A., Clamp, M. \& Barton, G. J. Jalview Version 2-a multiple sequence alignment editor and analysis workbench.

Bioinformatics 25, 1189-1191 (2009).

40. Saha, C. K., Sanches Pires, R., Brolin, H., Delannoy, M. \& Atkinson, G. C. FlaGs and webFlaGs: discovering novel biology through the analysis of gene neighbourhood conservation. Bioinformatics 37, 1312-1314 (2021). 
bioRxiv preprint doi: https//doi org/10.1101/2021.06 12 448176; this version posted November 4,2021 . The copyright holder for this preprint (which was not certified by peer review) is the author/funder, who has granted bioRxiv a license to display the preprint in perpetuity. It is made available under aCC-BY-ND 4.0 International license.

\section{Acknowledgements}

We thank Carlo de Oliveira Martins for proteomics analyses. We are also grateful to David Kysela for assistance with phylogenetic analysis, Phil Robinson for taking photographs of Streptomyces colonies, and the members of the JIC Bioimaging platform, including Elaine Barclay for help with electron microscopy and Sergio Lopez for support with image analysis. We thank Joseph W. Sallmen for helpful comments on the manuscript and $\mathrm{M}$. Thanbichler for the gift of HADA. This work was funded by the Royal Society (URF\R1\180075) to SS and by the BBSRC Institute Strategic Program grant BBS/E/J000PR9791 to the John Innes Centre.

\section{Author Contributions}

MJB and SS conceived the study, performed experiments and interpreted data, KAG performed phylogenetic analysis, GC conducted computational analyses, KCF performed electron microscopy experiments. SS supervised project. MJB and SS wrote the manuscript which was approved by all coauthors.

\section{Competing Interests}

The authors declare no competing interests. 


\section{Figures}

Figure 1

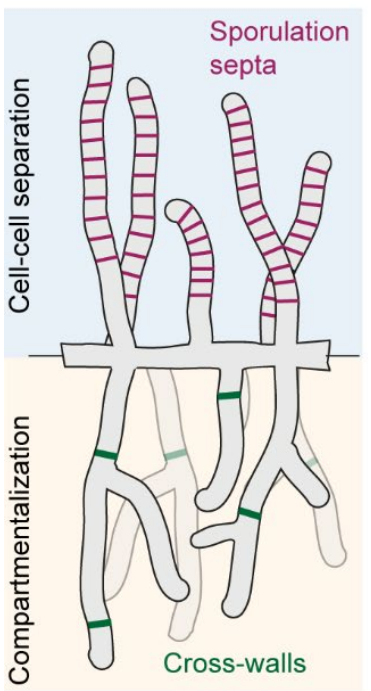

b
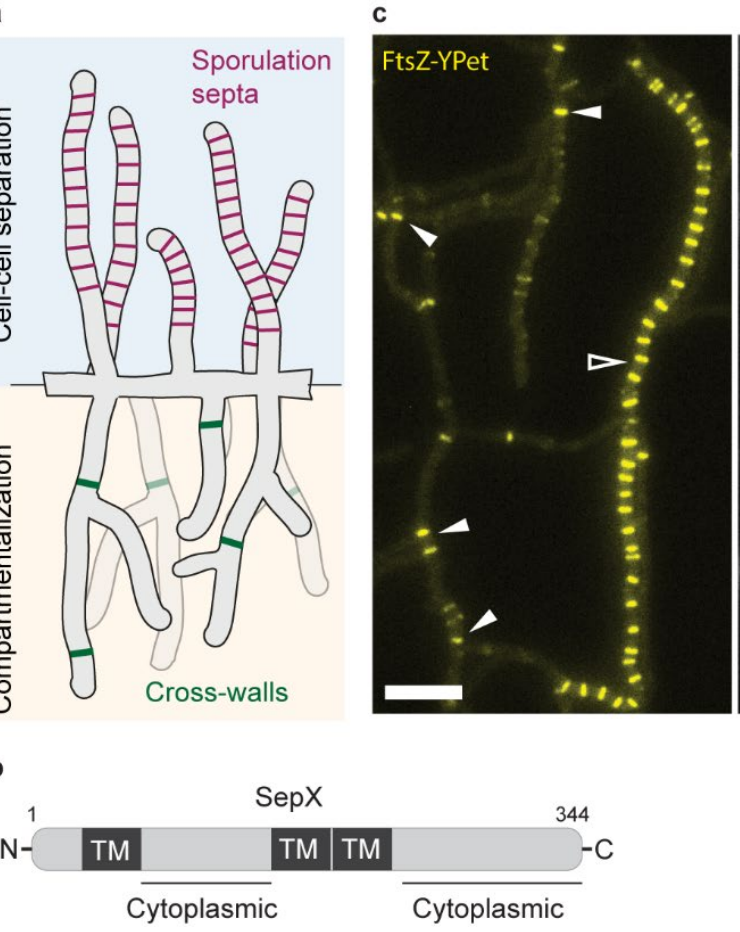

d

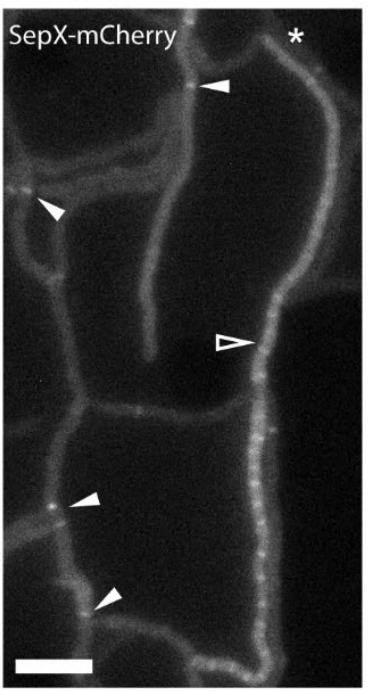

bar: 5 microns

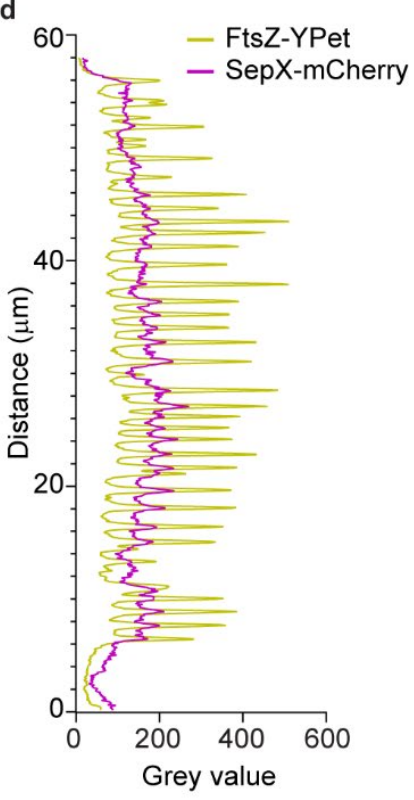

Frame $9(17)$

Figure 1: SepX co-localizes with FtsZ at cross-walls and sporulation septa. (a) Schematic showing the two kinds of division septa that are formed in Streptomyces: vegetative cross-walls (green) and sporulation septa (magenta). (b) Schematic depicting the predicted domain organization of SepX (vnz_14865), including three trans-membrane (TM) segments and two cytoplasmic loops. Numbers denote amino acid count. (c) Microscopic analysis of a merodiploid strain expressing both a SepXmCherry fusion and a FtsZ-YPet fusion (MB256) reveals that SepX and FtsZ co-localize at both crosswalls (filled arrows) and sporulation septa (open arrow). Shown is a single frame from Supplementary Movie 1. Experiments were performed in duplicate. Scale bar: $5 \mu \mathrm{m}$. (d) Intensity profiles of FtsZ-YPet and SepX-mCherry fluorescence extracted from the sporogenic hyphae $\left({ }^{*}\right)$ presented in panel (c). Source data are provided as a Source Data file. 

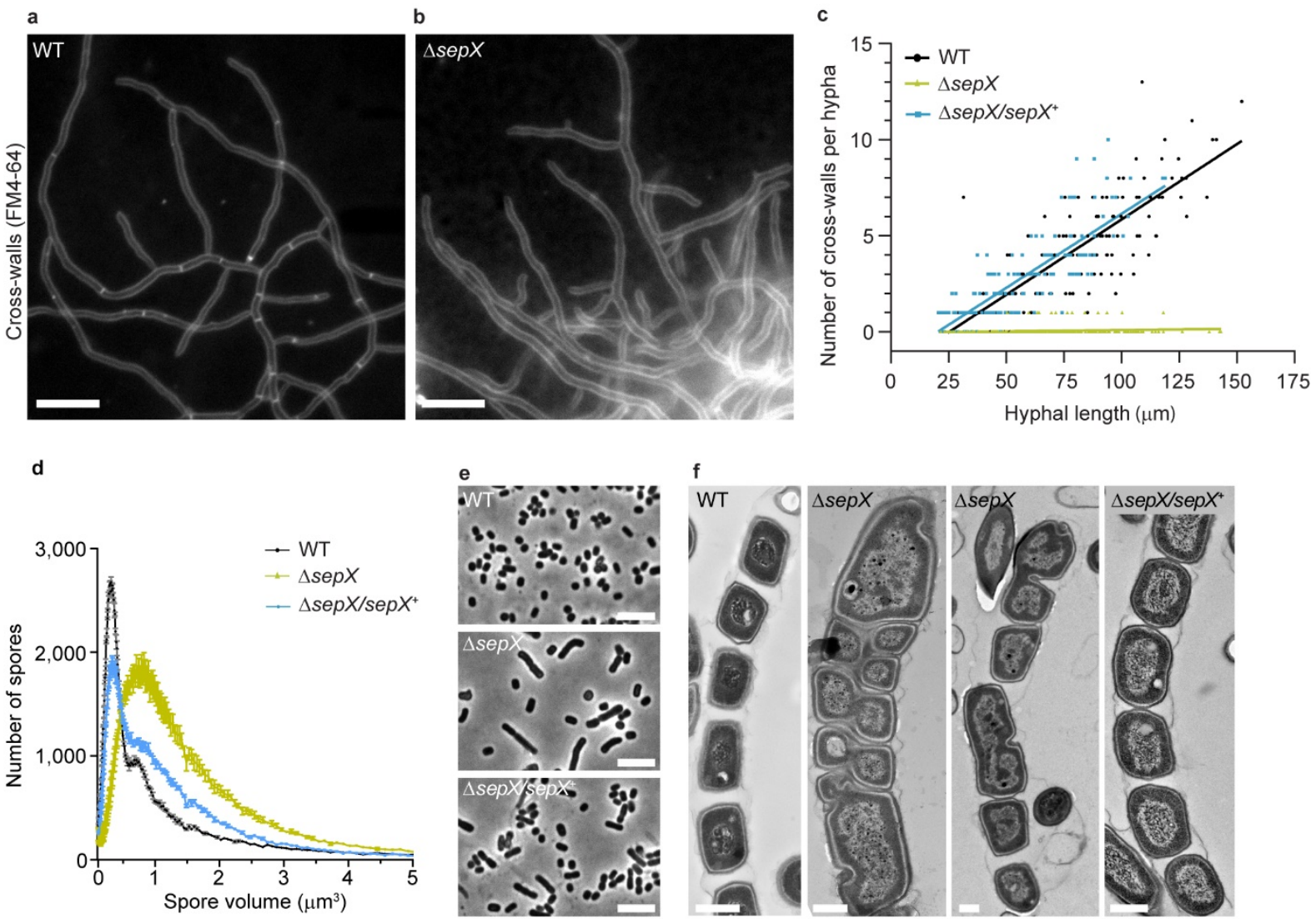

Figure 2: SepX is required for vegetative cross-wall formation and regular sporulation septation. (a) Vegetative septa can be visualized by fluorescence microscopy after incubating wild-type (WT) $S$. venezuelae hyphae with the membrane dye FM4-64. (b) The sepX deletion strain (SV55) lacks septa in growing hyphae, as visualized by FM4-64 staining. Scale bars in a and b: $10 \mu \mathrm{m}$. Data shown in (a) and (b) are representatives for experiments performed in triplicate. (c) Quantification of cross-wall frequency in hyphae emerging from spores of the wildtype (WT), the $\Delta$ sepX mutant (SV55) and the complemented mutant $\Delta \operatorname{sepX} / \operatorname{sep} X^{+}(\mathrm{MB} 745)$. Cross-walls were visualized by growing the strains in the presence of $0.25 \mathrm{mM} \mathrm{HADA}$ for 5-6 hours following germination. Data was derived from triplicate experiments and at least 20 spores per replicate were analyzed. Solid lines represent simple linear regression. (d) Spore volume frequency distributions (normalized to total count, $n=100,000$ ) of the wildtype (WT), the $\Delta$ sepX mutant (SV55) and the complemented mutant (MB181). Shown is the mean \pm s.e.m. of three biological replicates per strain. (e) Representative phase contrast images of spores analyzed in (d). Data shown are representatives for experiments performed in triplicate. Scale bars: 5 $\mu \mathrm{m}$. (f) TEM images of sporulating hyphae in the wildtype (WT), the $\triangle$ sepX mutant (SV55) and the complemented mutant $\Delta \operatorname{sepX} / \operatorname{sep} X^{+}$(MB181). Scale bars: $500 \mathrm{~nm}$. Shown are representative images of at least 5 independent hyphae. Source data are provided as a Source Data file for (c) and (d). 
Figure 3

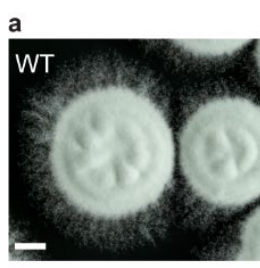

Sporulation Cross-walls

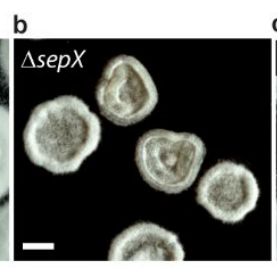

Y

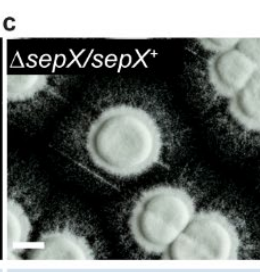

$Y$
$Y$

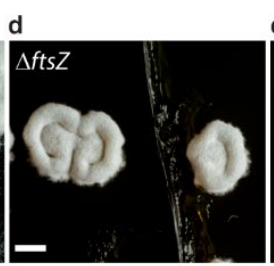

$N$

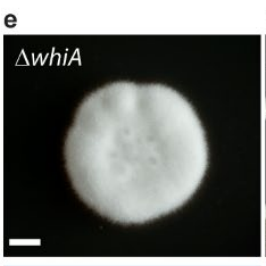

$\mathrm{N}$

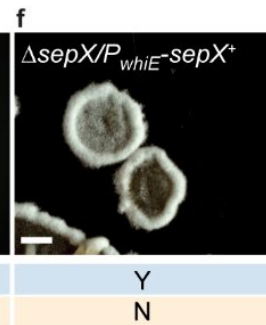

Figure 3: Cross-walls are crucial for cellular development of $\boldsymbol{S}$. venezuelae. Representative images of the colony morphology of (a) the wild-type (WT), (b) the $\Delta$ sepX mutant (SV55), (c) the complemented mutant (MB181), (d) the $\Delta f t s Z$ mutant (DU669), (e) the $\Delta$ whiA mutant (SV7), or (f) the conditional sepX mutant expressing sepX from the sporulation-specific whiE promoter (MB1120). All strains were grown on solid MYM and photographed after three days. " $\mathrm{Y}$ " (Yes) and " $\mathrm{N}$ " (No) indicate whether strains can form cross-walls and/or sporulation septa. Scale bars: $1 \mathrm{~mm}$. 
Figure 4
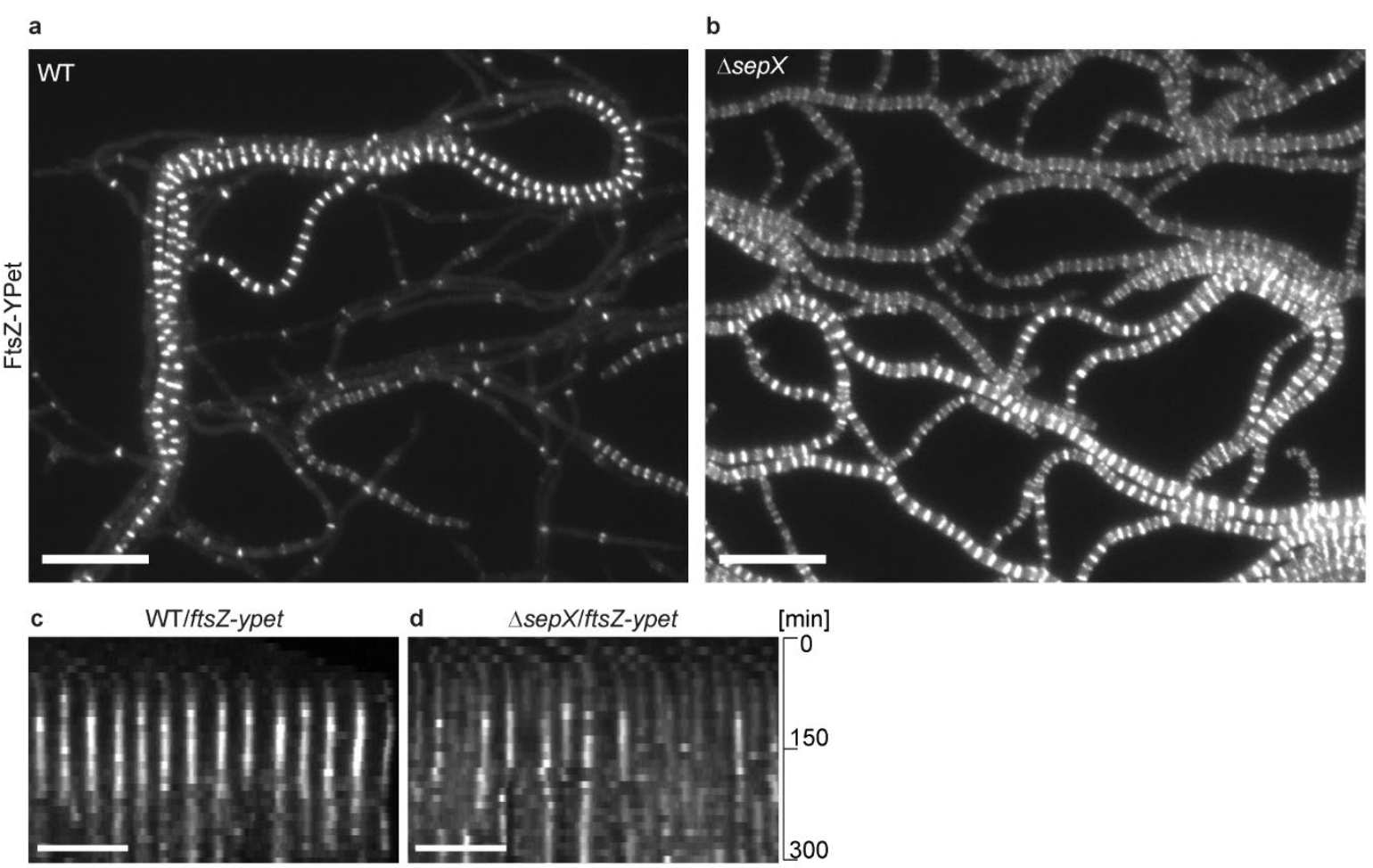

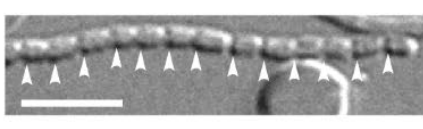

e

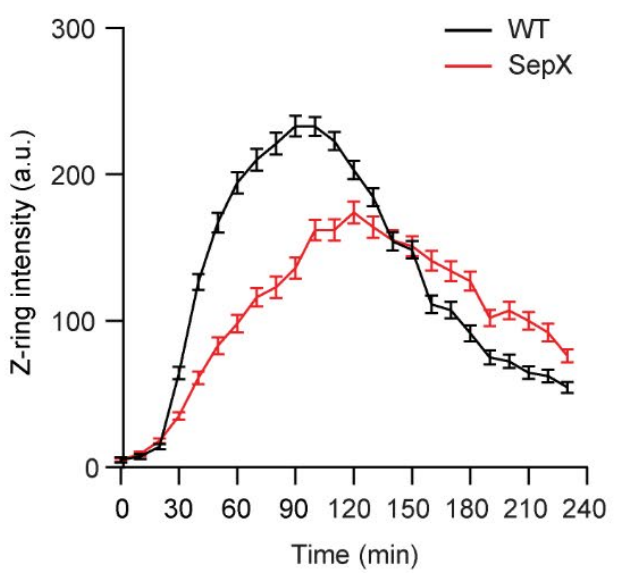

f

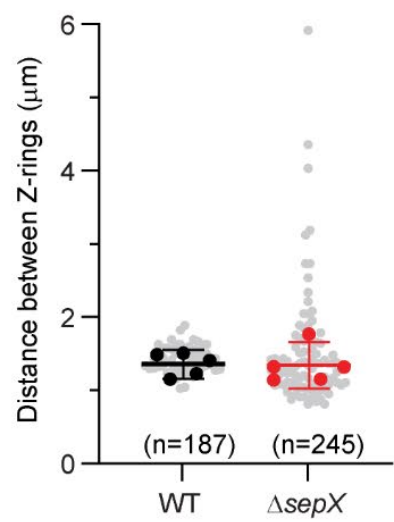

g

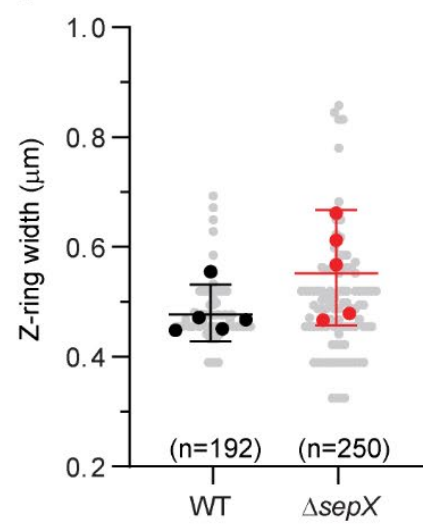

Figure 4: SepX is important for Z-ring stability and architecture during sporulation-specific cell division. Still images from Supplementary Movie 2 and 3 showing YPet-labelled Z-ring formation in sporulating hyphae of (a) the wildtype (SS12) or (b) the $\Delta$ sepX mutant (MB180). Scale bar: $10 \mu \mathrm{m}$. (c) and (d) Subsection of representative kymographs (top panel) showing the spatiotemporal localization of FtsZ-YPet in (c) the wildtype (WT, SS12) and (d) the $\Delta$ sepX mutant (MB180). Y-axis shows imaging duration. Bottom panel depicts terminal sporulation phenotype with filled arrow heads pointing at completed sporulation septa, open arrow heads at failed septation. Scale bars: $5 \mu \mathrm{m}$. Complete kymographs are shown in Supplementary Figure $4 \mathrm{~d}$ and e. (e) Fluorescence intensity traces of FtszYPet (Z-rings) over time derived from sporulating WT (SS12) and $\Delta$ sepX mutant hyphae (MB180). Shown are the mean fluorescence intensity traces (mean \pm s.e.m.) collected from Z-rings of five sporulating hyphae for each strain ( $n=5$ independent hyphae per strain). (f) Distance and (g) width of 
bioRxiv preprint doi: https://doi.org/10.1101/2021.06.12.448176; this version posted November 4, 2021. The copyright holder for this preprint (which was not certified by peer review) is the author/funder, who has granted bioRxiv a license to display the preprint in perpetuity. It is made available under aCC-BY-ND 4.0 International license.

Z-rings in sporulating hyphae of WT (SS12) and sepX-deficient hyphae (MB180). Analysis is based on the same data time-lapse data that was used to generate panel (e). Shown are the mean values for Zring distance and width for reach replicate (black and red dots) $\pm 95 \% \mathrm{Cl}$. $n$-number of Z-rings from five representative hyphae per strain were analyzed. The data shown here are representative for experiments performed in duplicate. Source data are provided as a Source Data file for (e)-(g). 
Figure 5

a

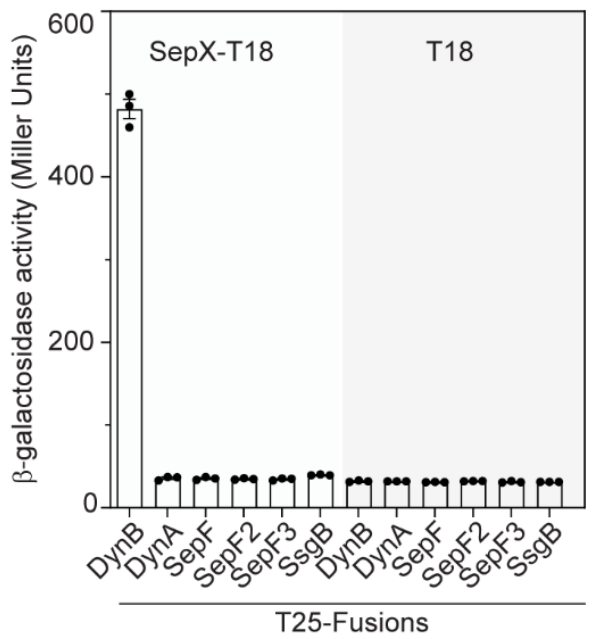

c

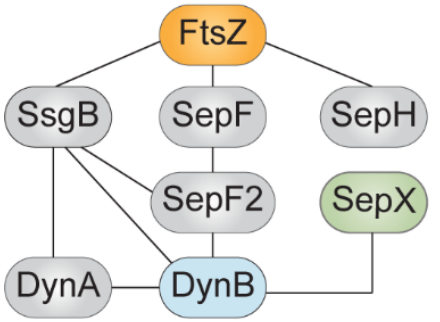

Streptomyces divisome

b

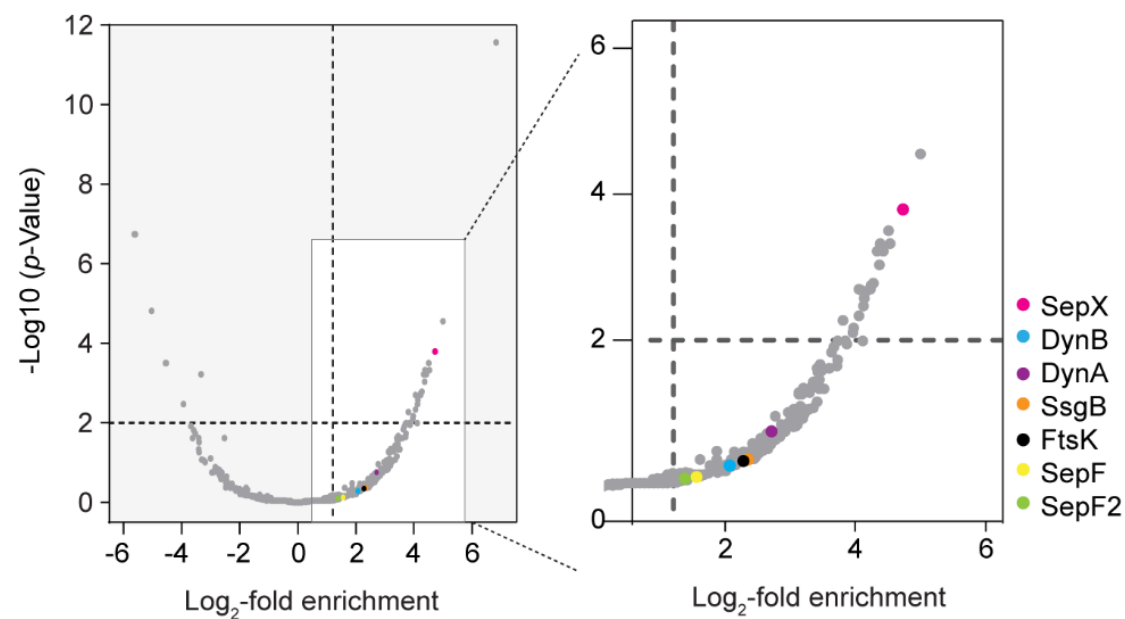

Figure 5: SepX is part of the S. venezuelae divisome and specifically interacts with DynB. (a) Bacterial two-hybrid analysis and corresponding $\beta$-galactosidase activities between SepX-T18 or the empty plasmid (T18) and the indicated T25-fusions. Data points present mean values +/- s.e.m. Each interaction was assayed in biological triplicate. (b) Co-immunoprecipitation and mass spectrometry analysis of SepX. Immunoprecipitation experiments were performed using the control strain SS92 (dynB-ypet) or a strain expressing a sepX-FLAG/dynB-ypet fusion (MB942) and anti-Flag antibodyconjugated magnetic beads. Proteins that were retained on the beads were analyzed using mass spectrometry. Data obtained from three biological replicate experiments are displayed as a volcano plot (left) with several putative interaction partners highlighted in the enlarged subsection of the plot (right). The complete data set is included in the Source Data. (c) Schematic showing the proposed SepX interaction network, including DynB and several known S. venezuelae divisome components ${ }^{11,12}$. 
bioRxiv preprint doi: https://doi.org/10.1101/2021.06.12.448176; this version posted November 4, 2021. The copyright holder for this preprint (which was not certified by peer review) is the author/funder, who has granted bioRxiv a license to display the preprint in perpetuity. It is made available under aCC-BY-ND 4.0 International license.

Figure 6

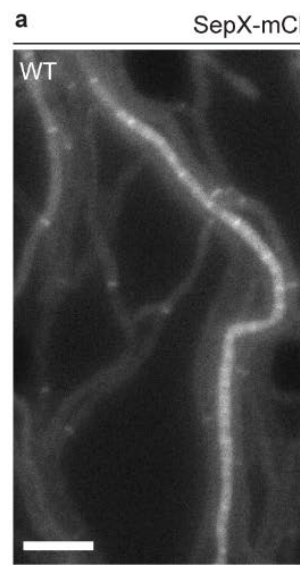

SepX-mCherry

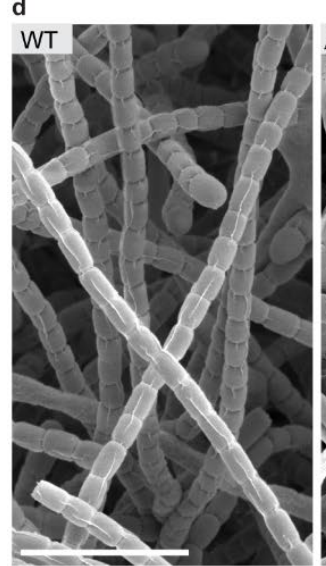

g

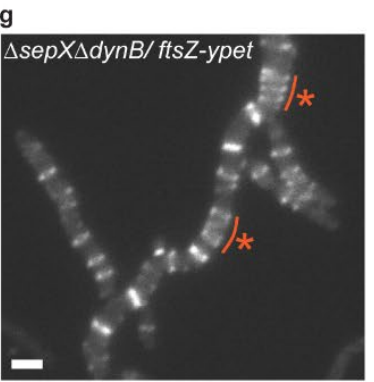

b
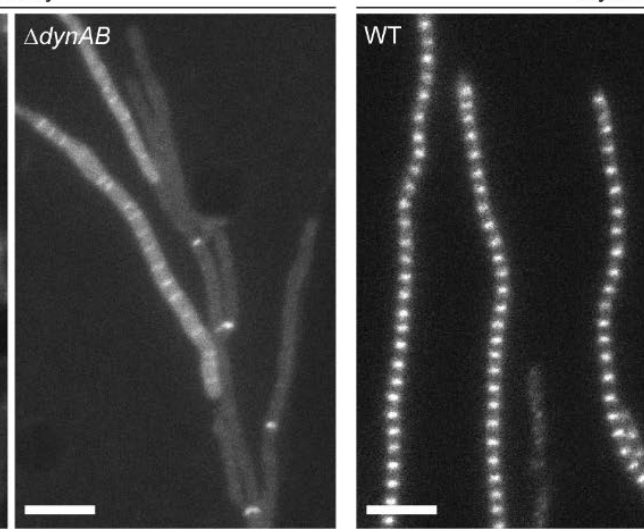

DynB-YPet

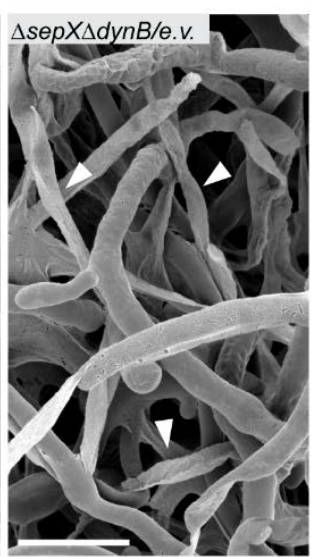

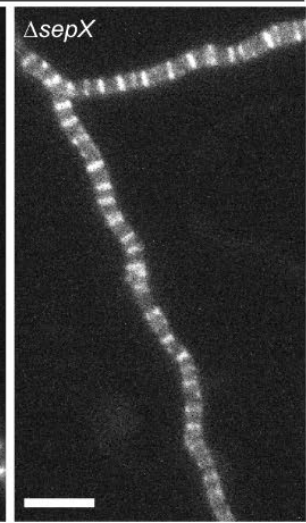
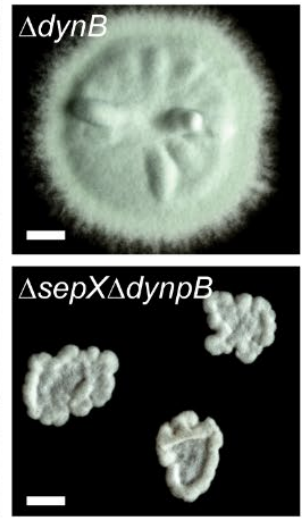

f

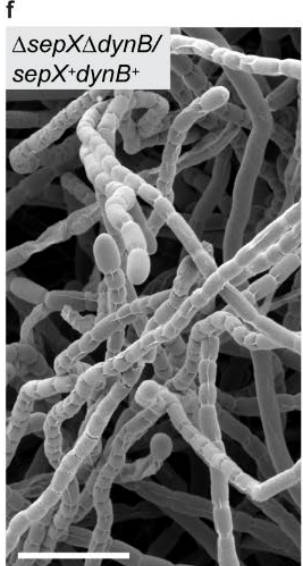

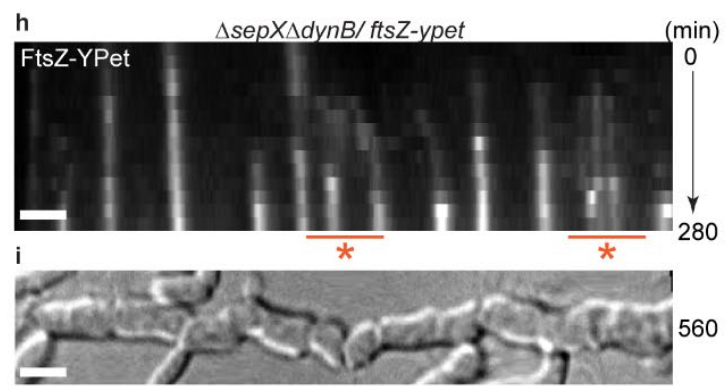

Figure 6: SepX and DynB function independently during sporulation but cause a synthetic sporulation defect when deleted together. (a) and (b) Microscopic analysis showing the subcellular localization of SepX-mCherry or DynAB-YPet in the wildtype (MB256, SS92) or in the absence of either dynAB (MB1270) or sepX (SS155). Data shown are representatives for experiments performed in duplicate. Scale bars: $5 \mu \mathrm{m}$. (c) Colony morphology of the $\Delta d y n B$ mutant (SS2) compared to the $\Delta$ sepX $\Delta d y n B$ double mutant (SV57). Strains were grown for 3 days on solid MYM. Scale bar: $1 \mathrm{~mm}$. (d)(f) Cryo-SEM images of $S$. venezuelae sporogenic hyphae, including (d) the wildtype (WT) and the $\Delta$ sepX $\Delta d y n B$ double mutant carrying an empty plasmid (MB1099), white arrow heads in right panel point at lysed hyphae, (e) the $\Delta \operatorname{sepX} \Delta d y n B$ double mutant complemented with either sepX (MB1102) or $d y n B$ (MB1101) expressed from the $e r m E^{*}$ promoter (+), or (f) the $\Delta \operatorname{sep} X \Delta d y n B$ double mutant complemented with both sepX and $d y n B$ (MB1103). Scale bars: $5 \mu \mathrm{m}$. Data shown in (d)-(f) are representatives of at least seven images. (g) Still images taken from Supplementary Movie 3 showing 
bioRxiv preprint doi: https://doi.org/10.1101/2021.06.12.448176; this version posted November 4, 2021. The copyright holder for this preprint (which was not certified by peer review) is the author/funder, who has granted bioRxiv a license to display the preprint in perpetuity. It is made available under aCC-BY-ND 4.0 International license.

FtsZ-YPet localization in the $\Delta s e p X \Delta d y n B$ mutant (MB1111). Orange line and asterisk denote regions of migrating Z-rings. Scale bar: $2 \mu \mathrm{m}$. (h) and (i) Kymograph showing FtsZ-YPet distribution in a sporulation $\triangle \operatorname{sep} X \Delta d y n B$ hypha presented in (g) with the corresponding DIC image (i) displaying the terminal sporulation phenotype. Orange line and asterisk in $(\mathrm{h})$ correspond to the region highlighted in (g). Scale bars: $2 \mu \mathrm{m}$. Data shown in (g)-(i) are representatives for experiments performed in duplicate. 


\section{Figure 7}

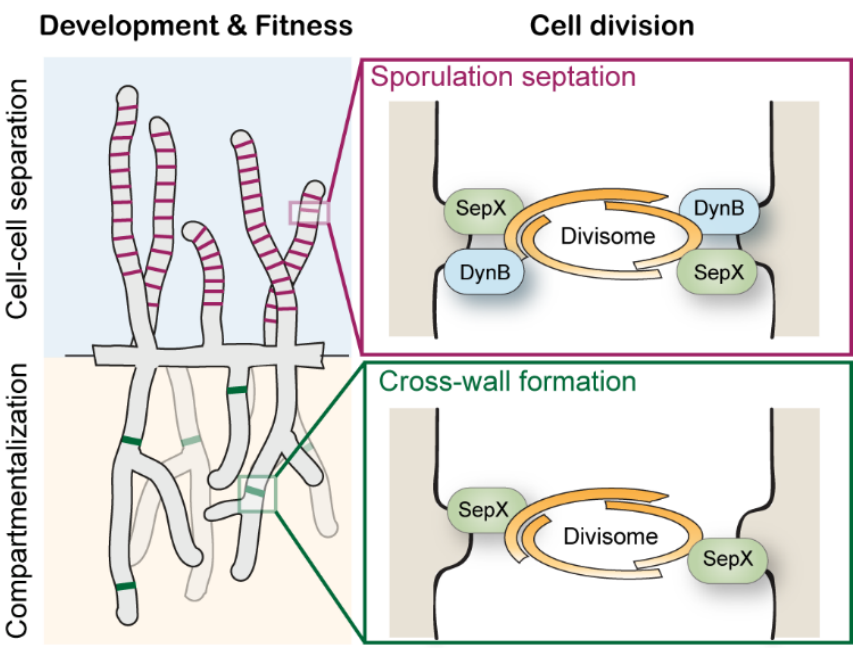

Figure 7: Proposed model for SepX function in Streptomyces cell division. During vegetative growth, SepX is crucial for the establishment of stable Z-rings leading to cross-wall formation and the compartmentalization of the hyphae which in turn is required for the coordinated progression through the life-cycle and overall fitness of the organism. During reproductive growth, SepX functions together with the dynamin-like protein DynB to ensure the spatial confinement of Z-rings, thereby stabilizing divisome assembly, function and efficient sporulation septation. 